\title{
Plástica da valva mitral em pacientes consecutivos. Como é a evolução tardia?: avaliação clínica e ecocardiográfica
}

Pablo M. A. POMERANTZEFF*, José G. AZEVEDO*, Miguel RATTI*, Álvaro V. MORAES*, Marisa AMATO*, Max GRINBERG*, Ana C. MONTEIRO*, Luiz B. PUIG*, Noedir A. G. STOLF*, Geraldo VERGINELLI*, Adib D. JATENE*

POMERANTZEFF, P. M. A.; AZEVEDO, J. G.; RATTI, M.; MORAES, A. V.; AMATO, M.; GRINBERG, M.; MONTEIRO, A. C.; PUIG, L. B.; STOLF, N. A. G.; VERGINELLI, G.; JATENE, A. D. - Plástica da valva mitral em pacientes consecutivos. Como é a evolução tardia?: avaliação clínica e ecocardiográfica. Rev. Bras. Cir. Cardiovasc., 6(2): 63-79, 1991.

RESUMO: Foram estudados no pós-operatório tardio 39 pacientes submetidos a plástica da valva mitral por insuficiência ou dupla lesão. A idade média dos pacientes foi de 30,5 anos com desvio padrão de 17,2 anos sendo $24(61,50 \%)$ pacientes do sexo feminino e $15(38,50 \%)$ do sexo masculino. Foram realizadas 21 anuloplastias com anel de Carpentier, com tira posterior, cinco anuloplastias tipo Merendino, e uma anuloplastia tipo Kay. Não houve óbito imediato neste grupo de pacientes. Vinte e três $(58,97 \%)$ pacientes apresentavam em sua história sintomas relacionados a febre reumática, $12(30,76 \%)$ pacientes com etiologia não definida e quatro $(10,25 \%)$ com degeneração mucóide. O tempo de evolução foi de 1497 meses/pacientes, com média de 38,39 meses e desvio padrão de 16,08 meses. No período de pós-operatório tardio $34(87,74 \%)$ pacientes estavam em classe funcional I (NYHA) sendo que ocorreram dois $(5,12 \%)$ óbitos tardios e dois $(5,12 \%)$ pacientes foram reoperados. As taxas linearizadas dos eventos reoperação e tromboembolismo foram respectivamente de $1,6 \%$ e $0,8 \%$ por paciente/ano. A estimativa da função de sobrevida é de $94,87 \%$. Na avaliação ecocardiográfica comparando-se os valores pré e pós-operatórios encontramos diminuiçāo significativa do diâmetro diastólico do ventrículo esquerdo $(p=0.0001)$, do diâmetro sistólico do ventrículo esquerdo $(p=0.0001)$, e do diâmetro do átrio esquerdo $(p=0.0001)$. 0 estudo ecodopplercardiográfico pós-operatório demonstrou não haver alteração da área valvar ao esforço. $\mathrm{A}$ análise dos dados permite concluir que os pacientes submetidos a plástica da valva mitral apresentam estimativa de sobrevida e evolução clínica satisfatórias.

DESCRITORES: valva mitral, plastia.

\section{INTRODUÇĀO}

Várias são as doenças que afetam as valvas cardiacas, produzindo lesōes essencialmente mecânicas ${ }^{28.88}$.

A insuficiência valvar mitral se faz através de alteraçōes morfológicas dos diversos elementos valvares sen- do quase sempre acompanhada de dilataçāo da porção posterior do anel.

Utiliza-se o termo plástica da valva mitral ou reconstrução da valva mitral quando é realizado procedimento cirúrgico conservador em valvas que apresentam refluxo, isto é dupla lesāo ou insuficiência. Várias são as técnicas

Trabalho realizado no Instituto do Coração do Hospital das Clinicas da Faculdade de Medicina da Universidade de São Paulo. São Paulo, SP. Brasil.

Apresentado ao 18: Congresso Nacional de Cirurgia Cardiaca. Rio de Janeiro, RJ, 5 e 6 de abril, 1991.

* Do Instituto do Coraçâo do Hospital das Clínicas da Faculdade de Medicina da Universidade de São Paulo.

Endereço para separatas: Pablo Pameranztzeff. Av. Dr. Enéas Carvalho de Aguiar, 44. Divisão Cirúrgica. 05403 São Paulo, SP, Brasil. 
POMERANTZEFF, P. M. A.; AZEVEDO, J. G.; RATTI, M.; MORAES, A. V.; AMATO, M.; GRINBERG, M.; MONTEIRO, A. C.; PUIG, L. B.; STOLF, N. A. G.; VERGINELLI, G.; JATENE, A. D. - Plástica da valva mitral em pacientes consecutivos. Como é a evolução tardia?: avaliação clínica e ecocardiográfica. Rev. Bras. Cir. Cardiovasc., 6(2): 63-79, 1992.

que podem ser utilizadas na reconstrução mitral, destacando-se as anuloplastias. Em nosso meio os pacientes atingem muitas vezes um grau avançado de lesão sendo necessário na maioria deles, para a realização de uma plástica adequada, a escolha de um tipo de anuloplastia, com ou sem anel protético, além de técnicas associadas, como por exemplo, encurtamento de cordas tendíneas.

$\mathrm{Na}$ literatura, os primeiros relatos referem-se a soluçōes para valvas estenóticas, sugerindo-se as tentativas em valvas insuficientes.

As primeiras tentativas cirúrgicas para correção da insuficiência mitral foram feitas por HARKEN et alii ${ }^{52}$, DAVILA et alii ${ }^{30}$, BAILEY et alii ${ }^{9}$ e NICHOLS et alii ${ }^{73}$, entre outros.

Sob visão direta, em 1957, LILLEHEI et alii ${ }^{68}$ propuseram a assim chamada anuloplastia simétrica, já que os autores propunham a plicatura junto às comissuras, avançando na mesma proporção para a parte anterior e posterior do anel mitral.

Em nosso meio, JATENE* relata anuloplastia assimétrica com plicatura do anel posterior ancorado em tira de Teflon.

PENTHER ${ }^{75}$, em seu editorial na "Presse Medicale", 1969, chama a atençăo para o fato de que, quando săo realizadas plásticas da mitral, devam ser mantidos todos os componentes da mesma, podendo isto colaborar, de alguma forma, para a manutenção de função ventricular esquerda adequada.

Neste mesmo ano, CARPENTIER ${ }^{16}$ publica nova técnica de anuloplastia assimétrica com a utilização de um anel protético rígido. Neste trabalho, o autor destaca que algumas valvopatias mitrais não prejudicam totalmente a flexibilidade das cúspides, sendo a insuficiência causada principalmente devido à dilatação do anel mitral. $O$ autor afirma que o anel proposto corrige a valva através de remodelaçăo comissural e da cúspide anterior, além da redução do anel da cúspide posterior por plicaturas seriadas. Sem dúvida, nesta época o interesse pelas plásticas mitrais foi renovado.

Vários autores se interessaram em reparar cordas tendíneas destacando-se FRATER et alii ${ }^{39}$, que relatam cordas tendíneas confeccionadas com pericárdio bovino conservado em glutaraldeído, e GREGORI et alii ${ }^{45}$, em nosso meio, que criam novas cordas a partir da própria cúspide.

Sem dúvida, para conseguirmos bons resultados na reconstrução mitral, é necessário um bom conhecimento da anatomia da valva, e a observação da mesma pelo cirurgião no ato operatório deverá ser cuidadosa, estudando-se sistematicamente todas as suas estruturas (Figura 1).

\footnotetext{
* JATENE, A. D. - Comunicação pessoal.
}

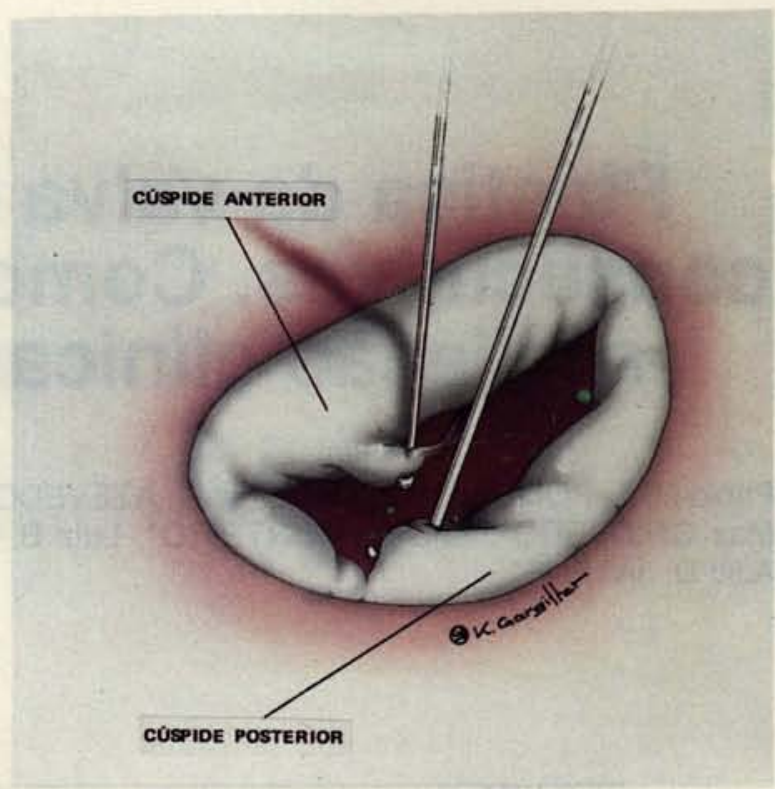

Fig. 1 - Tração nas cordas para avaliar prolapso ou retração.

O objetivo do nosso trabalho é estudar os resultados tardios do tratamento cirúrgico dos pacientes portadores de insuficiência mitral ou dupla lesão mitral com insuficiência predominante, os quais foram submetidos a plástica da valva mitral.

Para cumprir tal objetivo são analisados e comparados dados clínicos e laboratoriais pré e pós-operatórios.

\section{CASUÍSTICA E MÉTODOS}

Foram estudados 39 pacientes portadores de insuficiência ou dupla lesão mitral, lesōes estas isoladas ou associadas a outras lesōes cardiacas, adquiridas ou congênitas, e submetidos a plástica de valva mitral, no período de outubro de 1982 a outubro de 1987. Todos os pacientes foram operados pelo mesmo cirurgião. Esta série representa $95,12 \%$ do total de 41 plásticas realizadas no referido período.

Dos dois pacientes não analisados, um não tem seguimento atualizado no último ano e o outro foi uma reoperação (nova plástica). Não houve óbito imediato neste grupo de pacientes.

As lesōes valvares foram as seguintes: dupla lesão mitral - $12(30,76 \%)$ casos; insuficiência mitral - sete $(17,94 \%)$ casos; insuficiência mitral e aórtica - quatro $(10,25 \%)$ casos; dupla lesão mitral e insuficiência aórtica — três $(7,69 \%)$ casos; dupla lesão mitral e insuficiência tricúspide - um $(2,56 \%)$ caso; dupla lesão mitral e dupla 
POMERANTZEFF, P. M. A.; AZEVEDO, J. G.; RATTI, M.; MORAES, A. V.; AMATO, M.; GRINBERG, M.; MONTEIRO, A. C.; PUIG, L. B.; STOLF, N. A. G.; VERGINELLI, G.; JATENE, A. D. - Plástica da valva mitral em pacientes consecutivos. Como é a evolução tardia?: avaliação clínica e ecocardiográfica. Rev. Bras. Cir. Cardiovasc., 6(2): 63-79, 1992.

lesão aórtica - um $(2,56 \%)$ caso; dupla lesão mitral e insuficiência coronária - um $(2,56 \%)$ caso; dupla lesão mitral, insuficiência aórtica e tricúspide - um $(2,56 \%)$ caso; insuficiência mitral e insuficiência em bioprótese aórtica - um $(2,56 \%)$ caso; dupla lesão mitral, aórtica e tricúspide - um $(2,56 \%)$ caso; insuficiência em plástica de mitral - um $(2,56 \%)$ caso; insuficiência mitral e tricúspide e comunicaçāo interatrial - um $(2,56 \%)$ caso. Vinte e três $(58,97 \%)$ pacientes deste grupo apresentavam em sua história sintomas relacionados a febre reumática, $12(30,76 \%)$ pacientes com etiologia não definida e quatro $(10,25 \%)$ com degeneração mucóide.

Foram realizadas 21 anuloplastias com anel de Carpentier, sendo que em um dos pacientes foi associada anuloplastia tipo Merendino. Os números dos anéis de Carpentier utilizados foram $28 \mathrm{em}$ um paciente, $30 \mathrm{em}$ oito, 32 em nove, 34 em três. Foram realizados 12 anuloplastias com tira posterior, cinco anuloplastias de Merendino e uma tipo anuloplastia de Kay.

Em cinco $(12,8 \%)$ casos, foi realizado encurtamento de cordas tendíneas, em dois $(5,12 \%)$ retirada de cálcio e em um $(2,56 \%)$ retirada de cordas secundárias curtas.

Correções cirúrgicas associadas a plástica da valval mitral foram realizadas em $17(43,58 \%)$ pacientes; plástica de "De Vega" em valva tricúspide - cinco casos; substituiçăo da valva aórtica por bioprótese de pericárdio bovino modelo InCor, sendo uma retroca - quatro casos; desbastamento da valva aórtica e comissurotomia tricúspide - um caso; revascularização do miocárdio com anastomose da artéria torácica interna esquerda para a artéria interventricular anterior - um caso; comissorotomia da valva aórtica - um caso; desbastamento e comissurotomia de valva aórtica - um caso; substituição da valva aórtica por prótese mecânica modelo Omnicarbon - um caso; desbastamento de valva aórtica e exploração da valva tricúspide - um caso; atrioseptoplastia e plástica de "De Vega" em valva tricúspide - um caso; plástica de valva aórtica (plicatura central de uma válvula) e plástica de "De Vega" na valva tricúspide - um caso.

A idade dos pacientes variou de 10 a 73 anos com média de 30,5 anos e desvio padrão de 17,2 anos, sendo a mediana de 24 anos. Pertencem ao sexo feminino $24(61,50 \%)$ pacientes e ao sexo masculino $15(38,50 \%)$ pacientes. Vinte e oito $(71,80 \%)$ são da raça branca e $11(28,20 \%)$ da raça negra.

O tempo de evolução foi de 1497 meses/pacientes, com uma média de 38,39 meses e desvio padrão de 16,08 meses.

No período de pós-operatório todos os pacientes foram submetidos a avaliação clínica, além de ser analisado o rítmo cardíaco pelo eletrocardiograma. Foi realizado exame ecocardiográfico em $33(97,14 \%)$ pacientes do total dos 35 pacientes avaliados no último ano. Estudo ecodopplercardiográfico foi realizado em 16 (45,71\%), pacientes em condições basais e com esforço. Estudo hemodinâmico em condiçōes basais foi feito em oito $(22,85 \%)$ pacientes e com esforço em cinco $(14,28 \%)$. Para facilidade de análise, os dados clínicos e laboratoriais do período pré-operatório são apresentados no capítulo de resultados.

A evolução dos pacientes foi estudada pela análise dos parâmetros clínicos, eletrocardiográficos, ecocardiográficos.

Para análise da atividade física, os pacientes foram estudados pela classe funcional (NYHS) ${ }^{15}$, de acordo com o aparecimento ou não de dispnéia durante os vários graus de atividade física.

Nos períodos pré e pós-operatório foram analisados os sopros sistólicos em área mitral e classificados de uma $(+)$ a três $(+++)$ cruzes de acordo com a intensidade.

Foram analisadas as causas de morte e construídas curvas de estimativa da função de sobrevida para os eventos morte, reoperação e morte/reoperação. Foram também calculadas as taxas linearizadas dos eventos morte, reoperaçāo e tromboembolismo.

Foi analisado o ritmo cardíaco através do eletrocardiograma.

Os estudos ecocardiográficos foram realizados com equipamento marca Aloka SSD-860 com transdutores de 2,5,3,5 e 5,0 megahertz. As imagens foram obtidas nos cortes transversal, apical e subcostal em vários planos sendo gravados no mínimo três ciclos cardíacos consecutivos sendo considerados os valores médios, excluindo-se as imagens não bem definidas.

Foi utilizado o ecocardiograma modos Uni (M) e bidimensional para aferir os diâmetros sistólico (DSVE) e diastólico (DDVE) do ventrículo esquerdo, diâmetro sistólico, do átrio esquerdo (DAE), a dimensão diastólica na raiz da aorta (DAo), e as espessuras diastólicas do septo interventricular (EDS) e da parede posterior do ventrículo esquerdo (EPD), medidas estas que foram expressas em centímetros. A função do ventrículo esquerdo foi avaliada pelo $\mathrm{D}$, expresso em percentagem, e calculada a partir da fórmula

$D \%=\frac{\text { DDVE }- \text { DSVE }}{\text { DDVE }} \times 100$

O aspecto qualitativo da valva mitral foi analisado com os modos $\mathrm{M}$ e bidimensional, observando a mobilidade, grau de restrição e espessamento das cúspides, grau, de calcificação, bem como as alteraçōes em cordoalha, músculos papilares e anel. 
POMERANTZEFF, P. M. A.; AZEVEDO, J. G.; RATTI, M.; MORAES, A. V.; AMATO, M.; GRINBERG, M.; MONTEIRO, A. C.; PUIG, L. B.; STOLF, N. A. G.; VERGINELLI, G.; JATENE, A. D. - Plástica da valva mitral em pacientes consecutivos. Como é a evolução tardia?: avaliaçāo clínica e ecocardiográfica. Rev. Bras. Cir. Cardiovasc., 6(2): 63-79, 1992.

Foram comparados os valores pré e pós-operatórios do DSVE, DDVE, D, DAE, EDS e EPD.

O exame ecodopplercardiográfico foi realizado no pós-operatório em condiçōes basal e após esforço.

A deteç̧ão e quantificação do refluxo mitral (RM) foi feita com os mapeamentos de fluxo a cores e com o Doppler pulsátil. O mapeamento de fluxo a cores foi obtido nos cortes paraesternal e apical quatro câmaras. A severidade da regurgitação mitral foi graduada usando o critério descrito por ABBASI et alii ${ }^{~}$. Por este método, o grau de regurgitação depende da localização e da área de distribuição de fluxo anormal dentro do átrio esquerdo. se nenhum fluxo anormal for detectado no átrio esquerdo, traduz ausência de insuficiência mitral. $\mathrm{O}$ átrio esquerdo foi dividido em quatro áreas iguais, paralelas ao anel mitral, e à severidade da regurgitação mitral graduada de I a IV (discreta, moderada, importante ou severa), dependendo da extensão máxima da área alcançada pelo jato dentro do átrio esquerdo.

O Doppler contínuo, obtido em posição apical, corte quatro câmaras, foi utilizado para a obtenção das velocidades máxima (V) e média ( $\bar{V})$. A V é a maior velocidade obtida e $\bar{V}$ é o resultado da planimetria da curva de fluxo. Os gradientes $(G)$ foram calculados pela equação de BERNOULLI simplificada ${ }^{52}$ : GP $=4 \times \mathrm{V} 2$, expresso em $\mathrm{mmHg}$, representando o gradiente de pico, e GM $=4 \times \overline{\mathrm{V}} 2$, expresso em $\mathrm{mmHg}$ representando o gradiente médio (GM).

A área valvar (AV) expressa em $\mathrm{cm}^{2}$ foi calculada a partir da fórmula:

$$
\mathrm{AV}=\frac{220}{\mathrm{PHT}}
$$

onde o PHT representa o "Pressure Half Time", obtida na curva de fluxo pelo tempo decorrente entre a $\bar{V} e$ $\mathrm{V}^{52}$.

No fluxo pulmonar, estudado em posição paraesternal com eixo longo do tronco pulmonar, foram estudados os tempos de aceleração (TA), de fluxo (TF) e suas relaçōes (TATF), sendo que TA e TF foram medidos em segundos.

Após o exame basal os pacientes foram submetidos a esforço isotônico, em bicicleta ergométrica, até atingirem a exaustão física, sendo realizado novo exame ecocardiográfico, utilizando-se a mesma metodologia que foi utilizada em condições basais. Dentro do possivel, ou seja, com o paciente em freqüência respiratória e cardíaca modificadas, o novo traçado foi gravado para análise.

\section{A análise estatística dos dados constou de:}

a) para as variáveis quantitativas: inicialmente, estabeleceu-se os valores mínimos e máximos ocorridos e calculou-se médias e desvios-padrão. Posteriormente, empregou-se o teste T-Student pareado, com o objetivo de avaliar as condiçōes pré e pós-cirurgia.

b) cálculo das taxas livres de eventos: foram considerados como eventos morte, reoperação e morte + reoperação. Utilizou-se para tanto o método do produto limite de Kaplan-Meier. Calculou-se também a taxa linearizada, definida como o número total de eventos dividido pela soma do tempo de seguimento de cada paciente ${ }^{10}$. $11,47,48$.

Todos os cálculos foram feitos utilizando-se o "software" SAS (Statistical Analysis System) ${ }^{92}$.

Para análise dos resultados estabeleceu-se o valor de $5 \%$ para nivel de significância.

\section{RESULTADOS}

Do ponto de vista de atividade física no período pré-operatório, pertenciam a classe funcional IV 11 $(28,20 \%)$ pacientes, $26(66,66 \%)$ à classe funcional III e dois $(5,12 \%)$ à classe funcional II (Gráfico 1 ).

Os dois pacientes operados em classe funcional II apresentaram embolia pré-operatória.

No período de pós-operatório tardio estavam em classe funcional I, $34(89,74 \%)$ pacientes, três $(7,69 \%)$

\section{GRÁFICO 1 \\ CLASSE FUNCIONAL.}

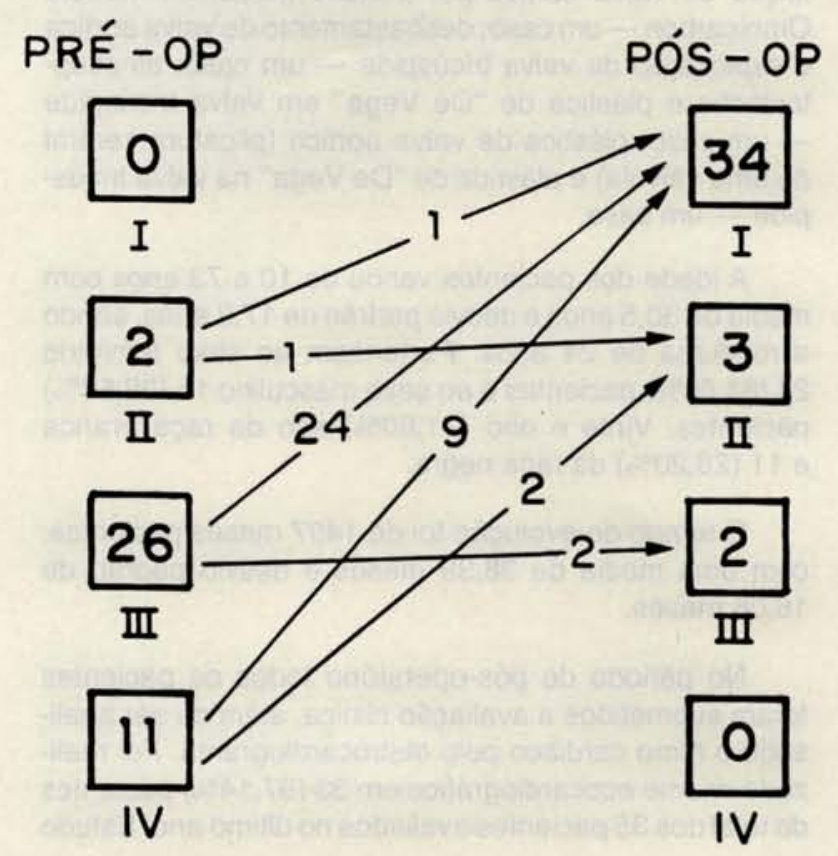


POMERANTZEFF, P. M. A.; AZEVEDO, J. G.; RATTI, M.; MORAES, A. V.; AMATO, M.; GRINBERG, M.; MONTEIRO, A. C.; PUIG, L. B.; STOLF, N. A. G.; VERGINELLI, G.; JATENE, A. D. - Plástica da valva mitral em pacientes consecutivos. Como é a evolução tardia?: avaliação clínica e ecocardiográfica. Rev. Bras. Cir. Cardiovasc., 6(2): 63-79, 1992.

em classe funcional II e dois $(8,64 \%)$ em classe funcional III. Nenhum paciente foi considerado em classe funcional IV no período de pós-operatório (Gráfico 1).

Dois $(5,12 \%)$ pacientes não obtiveram melhora após a operação, permanecendo na mesma classe funcional.

De $11(28,20 \%)$ pacientes em classe funcional IV no período pré-operatório, nove $(23,07 \%)$ tornaram-se assintomáticos e dois $(5,12 \%)$, apesar de melhora, evoluiram em classe funcional II.

Dos 26 pacientes $(66,66 \%)$ em classe funcional III no período pré-operatório, $24(61,53 \%)$ tornaram-se assintomáticos.

Dos dois $(5,12 \%)$ pacientes em classe funcional II no periodo pré-operatório, um $(2,56 \%)$ tornou-se assintomático, e o outro $(2,56 \%)$ permaneceu em classe funcional II (Gráfico 1).

No pré-operatório foi auscultado sopro sistólico com intensidade + + em $15(38,46 \%)$ pacientes, sendo que cinco $(12,82 \%)$ deixaram de apresentá-lo; oito $(20,51 \%)$ pacientes passaram a apresentá-lo com intensidade + e dois $(5,13 \%)$ pacientes continuam apresentando sopro de intensidade ++ .

Sopro sistólico com intensidade +++ foi auscultado em $18(46,16 \%)$ pacientes no pré-operatório, sendo que seis $(15,39 \%)$ deixaram de apresentá-lo; nove $(23,08 \%)$ pacientes passaram a apresentá-lo com intensidade + e três $(7,69 \%)$ pacientes com intensidade ++ .

Sopro sistólico com intensidade + foi auscultado em seis $(15,39 \%)$ pacientes, sendo que dois $(5,3 \%)$ continuaram de apresentá-lo com a mesma intensidade, três $(7,69 \%)$ tiveram aumento da intensidade para $++e$ um $(2,56 \%$ ) pacientes (caso 6 ) para +++ .

\section{GRÁFICO 2}

ESTIMATIVA DA FUNÇÄO DE SOBREVIDA DOS PACIENTES.

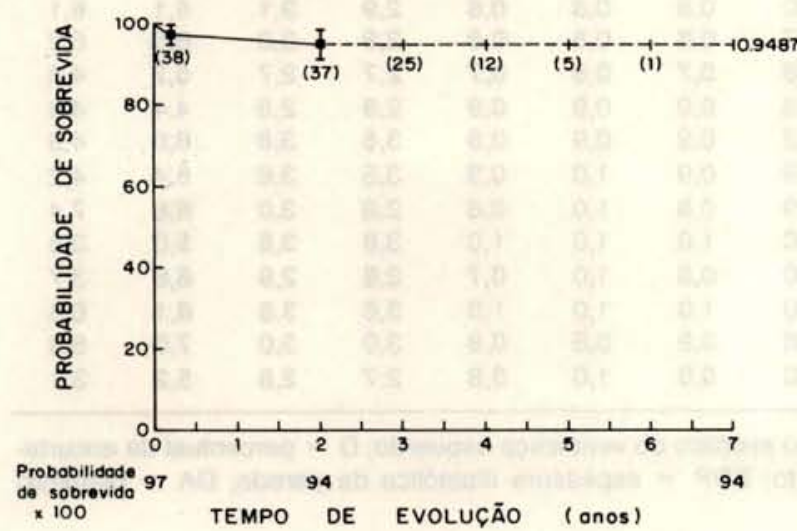

Dois $(5,12 \%)$ pacientes faleceram no período de pós-operatório tardio, sendo as causas de morte insuficiência hepática em um e deficiência enzimática congênita no outro (deficiência de enzima de conversão piruvato-kinase).

A taxa linearizada do evento morte foi e $1,6 \%$ por paciente/ano.

A estimativa da função de sobrevida dos pacientes é de $94,87 \%$ e desvio padrão de 3,53 (Gráfico 2 ).

Dois $(5,12 \%)$ pacientes foram reoperados no período de pós-operatório tardio respectivamente com 54 e 19 meses de evolução ambos com insuficiência cardíaca.

O paciente de número três foi submetido a substituição da valva mitral, plástica de valva-aortica e plástica

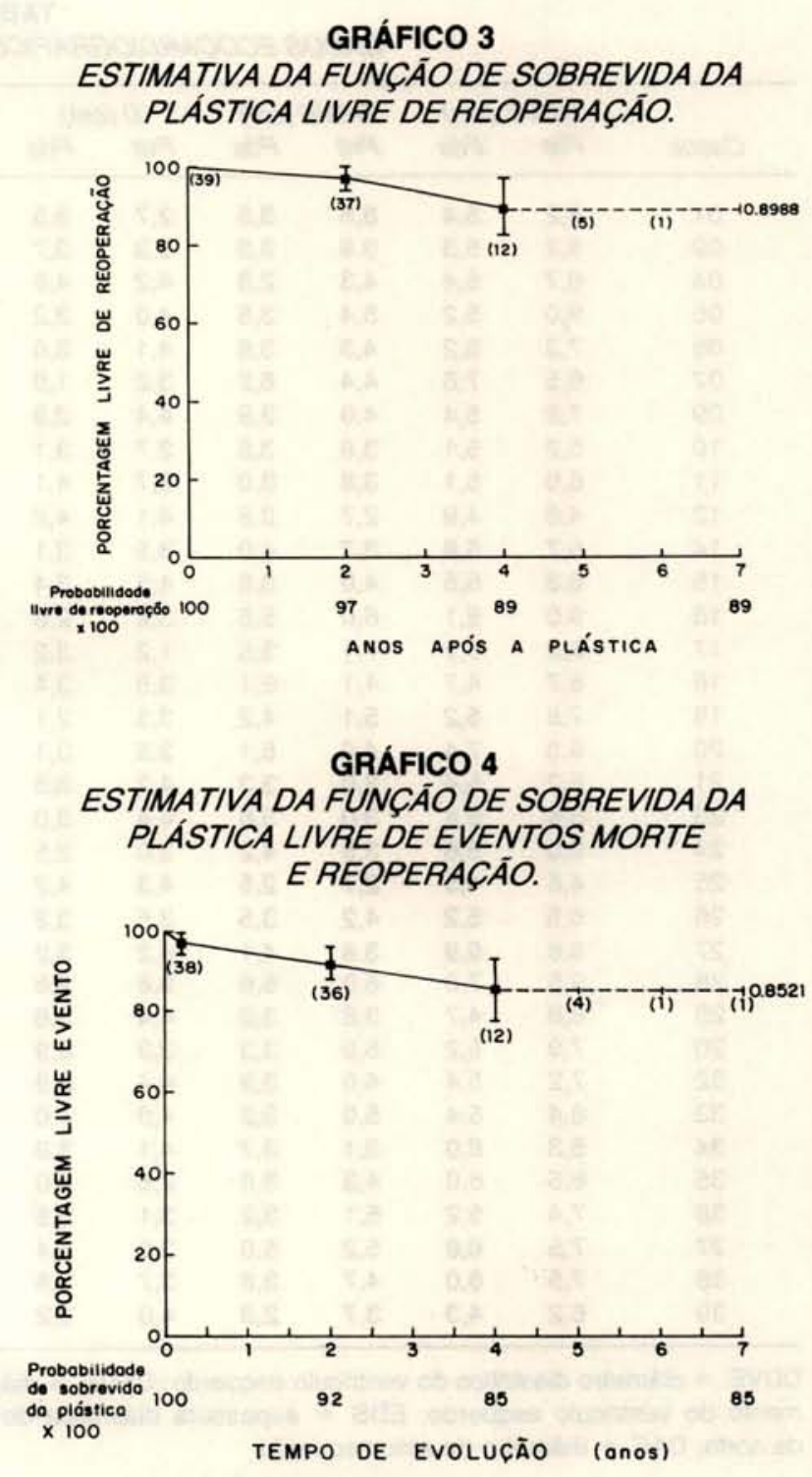


POMERANTZEFF, P. M. A.; AZEVEDO, J. G.; RATTI, M; MORAES, A. V.; AMATO, M.; GRINBERG, M.; MONTEIRO, A. C.; PUIG, L. B.; STOLF, N. A. G.; VERGINELLI, G.; JATENE, A. D. - Plástica da valva mitral em pacientes consecutivos. Como é a evolução tardia?: avaliação clínica e ecocardiográfica. Rev. Bras. Cir. Cardiovasc., 6(2): 63-79, 1992.

de "De Vega" da valva tricúspide em outro Serviço. O de número 12 foi por nós reoperado sendo realizada nova plástica da valva mitral.

A taxa linearizada do evento reoperação foi de 1,6\% por paciente/ano.

A estimativa da função de sobrevida da plástica livre de reoperação é de $89,88 \%$ e desvio padrão de 7,58 (Gráfico 3).

A estimativa da função de sobrevida da plástica livre de eventos morte e reoperação é de $85,21 \%$ e desvio padrāo de 7,88 (Gráfico 4).

A taxa linearizada de tromboembolismo no pós-operatório foi de $0,8 \%$ por paciente/ano.
Dezenove $(48,72 \%)$ pacientes que apresentavam rítmo sinusal no pré-operatório o mantiveram no pós-operatório; cinco $(12,82 \%)$ pacientes que apresentaram fibrilação atrial no pré-operatório o reverteram para rítmo sinusal; $13(33,33 \%)$ pacientes mantiveram no pós-operatório a fibrilaçāo atrial, já presente no pré-operatório; e dois $(5,13 \%)$ pacientes apresentavam ritmo sinusal no pré-operatório passaram a ter fibrilação atrial no pósoperatório.

Os valores ecocardiográficos pré e pós-operatórios encontram-se na Tabela 1 . Os resultados da análise estatística encontram-se na Tabela 2.

O valor máximo do diâmetro do ventrículo esquerdo (DDVE) no pré-operatório foi de $9,50 \mathrm{~cm}$, mínimo de $4,39 \mathrm{~cm}$, com média de $6,58 \mathrm{~cm}$ e desvio padrão de $1,33 \mathrm{~cm}$. No período de pós-operatório, o valor máximo

TABELA 1

DADOS ECOCARDIOGRÁFICOS PRÉ E POSS-OPERATÓRIOS.

\begin{tabular}{|c|c|c|c|c|c|c|c|c|c|c|c|c|c|c|}
\hline \multirow[b]{2}{*}{ Casos } & \multicolumn{2}{|c|}{ DDVE $(\mathrm{cm})$} & \multicolumn{2}{|c|}{ DSVE (cm) } & \multicolumn{2}{|c|}{$D(\mathrm{~cm})$} & \multicolumn{2}{|c|}{$E D S(\mathrm{~cm})$} & \multicolumn{2}{|c|}{$E D P(\mathrm{~cm})$} & \multicolumn{2}{|c|}{$D A(\mathrm{~cm})$} & \multicolumn{2}{|c|}{$D A E(\mathrm{~cm})$} \\
\hline & Pré & Pós & Pré & Pós & Pré & Pos & Pré & Pós & Pré & Pós & Pré & Pós & Pré & Pós \\
\hline 01 & 5,2 & 5,4 & 3,8 & 3,5 & 2,7 & 3,5 & 0,7 & 0,7 & 0,7 & 0,7 & 2,9 & 2,9 & 7,0 & 6,8 \\
\hline 02 & 5,7 & 5,3 & 3,8 & 3,3 & 3,3 & 3,7 & 0,9 & 1,0 & 0,9 & 1,0 & 2,8 & 2,8 & 7,2 & 6,3 \\
\hline 04 & 6,7 & 5,4 & 4,3 & 2,8 & 4,2 & 4,8 & 0,9 & 0,8 & 0,8 & 0,7 & 3,4 & 3,4 & 5,5 & 4,6 \\
\hline 05 & 9,0 & 5,2 & 5,4 & 3,5 & 4,0 & 3,2 & 1,2 & 1,1 & 0,9 & 1,1 & 2,9 & 2,9 & 6,5 & 5,0 \\
\hline 06 & 7,3 & 5,2 & 4,3 & 3,6 & 4,1 & 3,0 & 1,0 & 1,0 & 0,9 & 1,0 & 3,6 & 3,6 & 5,8 & 4,2 \\
\hline 07 & 6,5 & 7,8 & 4,4 & 6,2 & 3,2 & 1,0 & 1,1 & 1,1 & 1,1 & 1,1 & 3,4 & 3,5 & 6,3 & 6,2 \\
\hline 09 & 7,2 & 5,4 & 4,0 & 3,9 & 4,4 & 2,9 & 1,2 & 0,9 & 0,9 & 0,9 & 3,8 & 3,8 & 8,0 & 4,9 \\
\hline 10 & 5,2 & 5,1 & 3,8 & 3,5 & 2,7 & 3,1 & 8,8 & 0,8 & 0,8 & 0,9 & 2,2 & 2,5 & 5,5 & 4,2 \\
\hline 11 & 6,0 & 5,1 & 3,8 & 3,0 & 3,7 & 4,1 & 0,0 & 0,6 & 0,7 & 0,7 & 3,2 & 3,4 & 5,8 & 5,5 \\
\hline 12 & 4,6 & 4,9 & 2,7 & 2,8 & 4,1 & 4,2 & 0,8 & 0,7 & 0,8 & 0,7 & 3,3 & 3,0 & 5,0 & 5,2 \\
\hline 14 & 5,7 & 5,8 & 3,7 & 4,0 & 3,5 & 3,1 & 0,8 & 0,9 & 0,7 & 0,9 & 3,2 & 3,5 & 5,5 & 4,5 \\
\hline 15 & 6,3 & 5,5 & 4,0 & 3,6 & 4,5 & 3,4 & 0,9 & 1,1 & 1,1 & 1,0 & 2,6 & 2,6 & 4,8 & 3,5 \\
\hline 16 & 9,0 & 8,1 & 6,0 & 5,8 & 3,2 & 2,8 & 1,0 & 1,1 & 0,9 & 1,0 & 2,7 & 2,8 & 7,8 & 6,6 \\
\hline 17 & 6,2 & 5,2 & 7,1 & 3,5 & 1,2 & 3,2 & 1,1 & 1,1 & 1,0 & 1,1 & 3,7 & 3,8 & 5,2 & 4,6 \\
\hline 18 & 6,7 & 4,7 & 4,1 & 3,1 & 3,5 & 3,4 & 1,0 & 1,0 & 1,1 & 1,0 & 3,1 & 3,1 & 5,6 & 4,9 \\
\hline 19 & 7,8 & 5,2 & 5,1 & 4,2 & 3,3 & 2,1 & 1,0 & 0,9 & 1,1 & 0,9 & 2,5 & 2,9 & 7,7 & 5,8 \\
\hline 20 & 6,5 & 7,4 & 4,2 & 5,1 & 3,5 & 3,1 & 1,1 & 1,1 & 0,9 & 1,0 & 3,0 & 3,0 & 6,3 & 5,1 \\
\hline 21 & 5,2 & 5,4 & 3,0 & 3,3 & 4,2 & 3,8 & 1,3 & 1,0 & 0,9 & 1,0 & 3,3 & 3,6 & 5,0 & 3,9 \\
\hline 23 & 5,4 & 5,4 & 3,0 & 3,8 & 4,4 & 3,0 & 0,9 & 0,8 & 1,1 & 0,8 & 2,8 & 2,9 & 6,4 & 7,1 \\
\hline 24 & 5,0 & 5,6 & 3,5 & 4,2 & 3,0 & 2,5 & 1,0 & 0,9 & 0,7 & 0,9 & 3,6 & 3,7 & 5,3 & 5,3 \\
\hline 25 & 4,8 & 4,5 & 2,7 & 2,5 & 4,3 & 4,2 & 1,1 & 1,1 & 1,0 & 1,1 & 3,0 & 3,0 & 6,0 & 4.8 \\
\hline 26 & 6,5 & 5,2 & 4,2 & 3,5 & 3,5 & 3,2 & 0,7 & 0,7 & 0,7 & 0,7 & 2,2 & 2,2 & 5,5 & 4,2 \\
\hline 27 & 6,6 & 5,9 & 3,8 & 4,1 & 4,2 & 3,2 & 1,0 & 0,8 & 0,8 & 0,8 & 2,9 & 3,1 & 6,1 & 6,1 \\
\hline 28 & 9,5 & 7,6 & 6,0 & 5,6 & 3,6 & 2,6 & 0,7 & 0,8 & 0,8 & 0,8 & 2,9 & 3,0 & 8,8 & 6,2 \\
\hline 29 & 6,8 & 4,7 & 3,8 & 3,0 & 4,4 & 3,6 & 0,8 & 0,7 & 0,8 & 0,7 & 2,7 & 2,7 & 6,2 & 4,5 \\
\hline 30 & 7,9 & 5,2 & 5,0 & 3,3 & 3,3 & 3,9 & 0,8 & 0,9 & 0,9 & 0,9 & 2,9 & 2,8 & 4,4 & 4,0 \\
\hline 32 & 7,2 & 5,4 & 4,0 & 3,9 & 4,4 & 2,9 & 1,2 & 0,9 & 0,9 & 0,9 & 3,8 & 3,8 & 8,0 & 4,9 \\
\hline 33 & 8,4 & 5,4 & 5,0 & 3,2 & 4,0 & 4,0 & 0,9 & 0,9 & 1,0 & 0,9 & 3,5 & 3,6 & 6,4 & 4,2 \\
\hline 34 & 5,3 & 6,0 & 3,1 & 3,7 & 4,1 & 3,8 & 0,9 & 0,8 & 1,0 & 0,8 & 2,8 & 3,0 & 6,8 & 7,4 \\
\hline 35 & 6,5 & 6,0 & 4,3 & 3,6 & 2,6 & 4,0 & 1,0 & 1,0 & 1,0 & 1,0 & 3,8 & 3,8 & 5,0 & 3,8 \\
\hline 36 & 7,4 & 5,2 & 5,1 & 3,2 & 3,1 & 3,8 & 1,0 & 0,8 & 1,0 & 0,7 & 2,8 & 2,9 & 6,6 & 3,7 \\
\hline 37 & 7,5 & 6,8 & 5,2 & 5,0 & 3,0 & 2,4 & 1,0 & 1,0 & 1,0 & 1,0 & 3,6 & 3,6 & 8,1 & 6,6 \\
\hline 38 & 7,5 & 6,0 & 4,7 & 3,8 & 3,7 & 3,6 & 0,8 & 0,9 & 0,8 & 0,8 & 3,0 & 3,0 & 7,0 & 5,8 \\
\hline 39 & 6,2 & 4,3 & 3,7 & 2,9 & 4,0 & 3,2 & 1,0 & 0,9 & 1,0 & 0,8 & 2,7 & 2,8 & 5,2 & 3,1 \\
\hline
\end{tabular}

DDVE = diâmetro diastólico do ventrículo esquerdo; DSVE = diâmetro sistólico do ventrícuçp esquerdo; $\mathrm{D}=$ percentual de encurtamento do ventrículo esquerdo; EDS = espessura diastólica do septo; EDP = espessura diastólica da parede; $\mathrm{DA}=$ diâmetro da aorta; $\mathrm{DAE}=$ diâmetro de átrio esquerdo. 
POMERANTZEFF, P. M. A.; AZEVEDO, J. G.; RATTI, M.; MORAES, A. V.; AMATO, M.; GRINBERG, M.; MONTEIRO, A. C.; PUIG, L. B.; STOLF, N. A. G.; VERGINELLI, G.; JATENE, A. D. - Plástica da valva mitral em pacientes consecutivos. Como é a evolução tardia?: avaliação clínica e ecocardiográfica. Rev. Bras. Cir. Cardiovasc., 6(2): 63-79, 1992.

TABELA 2

VALOR MINIIMO. VALOR MÁXIMO E DESVIO PADRĀO DAS MÉDIAS, DAS VARIÁVEIS HEMODINANICAS NOS PERIODOS PRÉ E POSS-OPERATORIOS.

\begin{tabular}{|c|c|c|c|c|c|c|c|c|c|c|}
\hline Vaniável & & $\begin{array}{c}\text { No De } \\
\text { Pacientes }\end{array}$ & Condiçāo & $\begin{array}{c}\text { Valor } \\
\text { Mínimo }\end{array}$ & $\begin{array}{c}\text { Valor } \\
\text { Máximo }\end{array}$ & Média & $\begin{array}{l}\text { Desvio } \\
\text { Padrão }\end{array}$ & & $t$ & $P$ \\
\hline$D D V E$ & 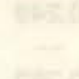 & 34 & $\begin{array}{l}\text { Pré } \\
\text { Pós }\end{array}$ & $\begin{array}{l}4,30 \\
4,30\end{array}$ & $\begin{array}{l}9,50 \\
8,10\end{array}$ & $\begin{array}{l}6,59 \\
5,60\end{array}$ & $\begin{array}{l}1,33 \\
0,93\end{array}$ & 45 & 4,55 & 0,0001 \\
\hline DSVE & taty & 34 & $\begin{array}{l}\text { Pré } \\
\text { Pós }\end{array}$ & $\begin{array}{l}2,50 \\
2,60\end{array}$ & $\begin{array}{l}7,20 \\
6,20\end{array}$ & $\begin{array}{l}4,21 \\
3,73\end{array}$ & $\begin{array}{l}1,02 \\
0,88\end{array}$ & & 2,77 & 0,0092 \\
\hline$D$ & $6=$ & 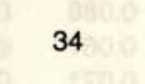 & $\begin{array}{l}\text { Pré } \\
\text { Pós }\end{array}$ & $\begin{array}{l}12,00 \\
20,00\end{array}$ & $\begin{array}{l}44,00 \\
48,00\end{array}$ & $\begin{array}{l}35,76 \\
33,73\end{array}$ & $\begin{array}{l}6,73 \\
6,43\end{array}$ & & 1,49 & 0,1440 \\
\hline$E D S$ & ther & 10 & $\begin{array}{l}\text { Pré } \\
\text { Pós }\end{array}$ & $\begin{array}{l}0,70 \\
0,60\end{array}$ & $\begin{array}{l}1,30 \\
1,10\end{array}$ & $\begin{array}{l}0,94 \\
0,89\end{array}$ & $\begin{array}{l}0,15 \\
0,15\end{array}$ & & 2,42 & 0,0213 \\
\hline$E D P$ & 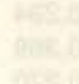 & 34 & $\begin{array}{l}\text { Pré } \\
\text { Pós }\end{array}$ & $\begin{array}{l}0,70 \\
0,70\end{array}$ & $\begin{array}{l}1,10 \\
1,10\end{array}$ & $\begin{array}{l}0,90 \\
0,88\end{array}$ & $\begin{array}{l}0,12 \\
0,14\end{array}$ & & 1,42 & 0,1630 \\
\hline$D A E$ & & 24 & $\begin{array}{l}\text { Pré } \\
\text { Pós }\end{array}$ & $\begin{array}{l}4,20 \\
3,10\end{array}$ & $\begin{array}{l}8,80 \\
7,40\end{array}$ & $\begin{array}{l}6,13 \\
5,07\end{array}$ & $\begin{array}{l}1,10 \\
1,12\end{array}$ & & 6,70 & 0,0034 \\
\hline
\end{tabular}

$\mathrm{t}=$ valor da estatística; $\mathrm{t}=$ Student; $\mathrm{p}=$ nível descritivo de probabilidade; DDVE = diâmetro diastólico do ventrículo esquerdo; DSVE = diâmetro sistólico do ventrículo esquerdo; $\mathrm{D}=$ percentual de encurtamento do ventrículo esquerdo; EDS = espessura diastólica do septo; EDP = espessura diastólica da parede; DAE = diâmetro do átrio esquerdo.

encontrado foi de $9,10 \mathrm{~cm}$, mínimo de $4.30 \mathrm{~cm}$, com média de $5,60 \mathrm{~cm}$ de desvio padrão de $0,93 \mathrm{~cm}$.

O DDVE diminuiu em $24(70,6 \%)$ pacientes, manteve-se em um $(2,9 \%)$ e aumentou em nove, $(26,5 \%)$ pacientes.

Houve uma redução significante $(p=0,0001)$ do DDVE quando comparados os valores pré e pós-operatórios.

O valor máximo do diâmetro sistólico do ventrículo esquerdo (DSVE) no pré-operatório foi de $7,20 \mathrm{~cm}$, mínimo de $2,50 \mathrm{~cm}$, com média de $4,21 \mathrm{~cm}$ e desvio padrão de $1,02 \mathrm{~cm}$. No período de pós-operatório o valor máximo foi de $6,20 \mathrm{~cm}$, mínimo de $2,60 \mathrm{~cm}$, com média de 3,72 cm e desvio padrão de $0,88 \mathrm{~cm}$.

O diâmetro sistólico do ventrículo esquerdo diminuiu em $24(70,6 \%)$ pacientes e aumentou em 10 (29.4\%) pacientes.

Comparando-se os valores pré e pós-operatórios, a reduçāo do DSVE foi significante ( $p=0,0092$ ).

O porcentual de encurtamento do ventrículo $D \%$ esquerdo diminuiu em $22(64,7 \%)$ pacientes, aumentou em $11(32,4 \%)$ pacientes e manteve-se em um $(2,9 \%)$ paciente (caso 33).

Comparando-se os valores obtidos no pré e pósoperatório, nāo há diferença significante ( $p=0,1440)$.

Com relação aos diâmetros de átrio esquerdo os valores máximo e mínimo no pré-operatório foram 8,80 $\mathrm{cm}$ e $4,20 \mathrm{~cm}$ respectivamente, com média de $6,13 \mathrm{~cm}$ de desvio padrão de $1,10 \mathrm{~cm}$.
No pós-operatório, o valor máximo foi de $7,40 \mathrm{~cm}$, o mínimo de $3.10 \mathrm{~cm}$, com média de $5,07 \mathrm{~cm}$ de desvio padrão de $1,12 \mathrm{~cm}$.

O diâmetro do átrio esquerdo diminuiu em 29 $(85,3 \%)$ pacientes, aumentou em três $(8,8 \%)$ e manteve-se em dois pacientes.

Houve redução significativa $(p=0,0001)$ comparando-se os valores pré e pós-operatórios.

$\mathrm{Na}$ Tabela 3, encontram-se os resultados obtidos nos exames ecodopplercardiográficos em condiçōes basal e no exercício, e na Tabela 4 encontram-se os resultados da análise estatística.

TABELA 3

DADOS ECODOPLERCARDIOGRÁFICOS EM CONDICOOES BASAIS E COM ESFORÇO (POSS-OPERATÓRIO

\begin{tabular}{lcr}
\hline Casos & $T A B / T F B$ & TAE/TFE \\
\hline 02 & 0,313 & 0,314 \\
07 & 0,365 & 0,271 \\
04 & 0,265 & 0,247 \\
16 & 0,426 & 0,567 \\
17 & 0,365 & 0,449 \\
18 & 0,498 & 0,346 \\
20 & 0,303 & 0,273 \\
24 & 0,363 & 0,312 \\
30 & 0,249 & 0,231 \\
33 & 0,407 & 0,264 \\
35 & 0,386 & 0,169 \\
36 & 0,472 & 0,423 \\
37 & 0,309 & 0,199 \\
39 & 0,436 & 0,256 \\
\hline
\end{tabular}


POMERANTZEFF, P. M. A.; AZEVEDO, J. G.; RATTI, M.; MORAES, A. V.; AMATO, M.; GRINBERG, M.; MONTEIRO, A. C.; PUIG, L. B.; STOLF, N. A. G.; VERGINELLI, G.; JATENE, A. D. - Plástica da valva mitral em pacientes consecutivos. Como é a evolução tardia?: avaliação clínica e ecocardiográfica. Rev. Bras. Cir. Cardiovasc., 6(2): 63-79, 1992.

TABELA 3

DADOS ECODOPLERCARDIOGRÁFICOS EM CONDIÇÓES BASAIS E COM ESFORÇO (PÓS-OPERATÓRIOS).

\begin{tabular}{|c|c|c|c|c|c|c|c|c|c|c|c|c|}
\hline Casos & $\begin{array}{c}G P B \\
(m m H g)\end{array}$ & $\begin{array}{c}\text { GPE } \\
(\mathrm{mm} H \mathrm{~g})\end{array}$ & $\begin{array}{c}G M B \\
(\mathrm{mmHg})\end{array}$ & $\begin{array}{c}G M E \\
(m m H g)\end{array}$ & $\begin{array}{l}A V B \\
\left(\mathrm{~cm}^{2}\right)\end{array}$ & $\begin{array}{l}A V E \\
\left(\mathrm{~cm}^{2}\right)\end{array}$ & $I M B$ & IMFE & $\begin{array}{l}T A B \\
\text { (seg) }\end{array}$ & $\begin{array}{l}\text { TAE } \\
\text { (seg) }\end{array}$ & $\begin{array}{l}\text { TFB } \\
\text { (seg) }\end{array}$ & $\begin{array}{c}\text { TFE } \\
\text { (seg) }\end{array}$ \\
\hline 01 & 15,0 & $19,96,6$ & 8,9 & 1,73 & 1,77 & + & + & - & - & - & - & \\
\hline 02 & 18,4 & 32,5 & 8,2 & 16,4 & 1,05 & 0,83 & 0 & 0 & 0,078 & 0,084 & 0,249 & 0,267 \\
\hline 04 & 12,4 & 14,9 & 4,1 & 4,9 & 3,27 & 3,13 & + & + & - & - & - & \\
\hline 07 & 20,0 & 31,7 & 9,6 & 13,4 & 2,26 & 2,16 & 0 & 0 & 0,092 & 0,063 & 0,252 & 0,232 \\
\hline 14 & 11,4 & 20,1 & 3,6 & 9,7 & 4,42 & 3,18 & + & + & 0,051 & 0,042 & 0,192 & 0,170 \\
\hline 16 & 11,0 & 27,0 & 5,0 & 10,7 & 2,39 & 1,85 & + & + & 0,124 & 0,088 & 0.291 & 0,155 \\
\hline 17 & 6,5 & 12,7 & 3,0 & 5,7 & 2,48 & - & 0 & 0 & 0,096 & 0,067 & 0,263 & 0,149 \\
\hline 18 & 10,4 & 17,1 & 7,2 & 8,5 & 2,42 & - & + & + &,- 134 & 0,071 & 0,269 & 0,205 \\
\hline 20 & 18,0 & 38,8 & 6,8 & 14,0 & 2,63 & 3,90 & + & + & 0,071 & 0,056 & 0,234 & 0,205 \\
\hline 24 & 11,8 & 17,0 & 4,8 & 8,4 & 2,09 & 2,09 & + & + & 0,085 & 0,071 & 0,234 & 0,227 \\
\hline 30 & 9,0 & 10,4 & 3,3 & 6,6 & 2,80 & 4,50 & + & + & 0,092 & 0,061 & 0,369 & 0.264 \\
\hline 33 & 7,2 & 15,6 & 4,9 & 7,6 & 3,34 & - & ++ & ++ & 0,110 & 0,060 & 0,270 & 0,227 \\
\hline 35 & 9,0 & 15,6 & 3,4 & 5,3 & 4,67 & - & + & + & 0,085 & 0,035 & 0,220 & 0,206 \\
\hline 36 & 7,7 & 8,1 & 4,1 & 6,8 & 3,69 & 4,20 & + & + & 0,121 & 0.103 & 0,256 & 0,243 \\
\hline 37 & 3,0 & 6,6 & 1,4 & 3,0 & 3,73 & 3,71 & 0 & 0 & 0,064 & 0,042 & 0,207 & 0,211 \\
\hline 39 & 9,5 & 11,0 & 5,9 & 14,0 & 3,50 & 3,96 & 0 & 0 & 0,099 & 0,049 & 0,227 & 0,191 \\
\hline
\end{tabular}

$\mathrm{B}=$ Condição basal; $\mathrm{E}=$ = Pós-esforço; $\mathrm{GP}=$ Gradiente de pico; $\mathrm{GM}=$ Gradiente Médio; $\mathbf{A V}=$ Área valvar mitral; $\mathrm{IM}=\mathrm{Grau}$ de regurgitação mitral; $0=$ Nenhuma regurgitação; $+=$ Insuficiência mitral discreta; $++=$ Insuficiência mitral moderada; TA

$=$ Tempode aceleração; TF $=$ Tempo de fluxo.

TABELA 4

VALOR MINIMO, VALOR MAXIMO E DESVIO PADRÃO DAS MÉDIAS, DAS VARIÁVEIS HEMODINÁNICAS NOS PERIODOS PRÉ E POSS-OPERATÓRIO.

\begin{tabular}{|c|c|c|c|c|c|c|c|c|}
\hline Variável & $\begin{array}{c}\text { N. De } \\
\text { Pacientes }\end{array}$ & Condiçâo & $\begin{array}{c}\text { Valor } \\
\text { Mínimo }\end{array}$ & $\begin{array}{c}\text { Valor } \\
\text { Máximo }\end{array}$ & Média & $\begin{array}{l}\text { Desvio } \\
\text { Padráo }\end{array}$ & $T$ & $P$ \\
\hline$G P$ & 16 & $\begin{array}{l}B \\
E\end{array}$ & $\begin{array}{l}3,00 \\
6,60\end{array}$ & $\begin{array}{l}20,00 \\
38,80\end{array}$ & $\begin{array}{l}11,26 \\
18,68\end{array}$ & $\begin{array}{l}4,63 \\
9,30\end{array}$ & 5,18 & 0,0001 \\
\hline$G M$ & 16 & $\vec{B}$ & $\begin{array}{l}1,40 \\
9,00\end{array}$ & $\begin{array}{r}9,60 \\
16,40\end{array}$ & $\begin{array}{l}5,12 \\
8,99\end{array}$ & $\begin{array}{l}2,14 \\
3,81\end{array}$ & $-3,04$ & 0,0081 \\
\hline$A V$ & 16 & B & $\begin{array}{l}1,05 \\
0,83\end{array}$ & $\begin{array}{l}4,67 \\
4,50\end{array}$ & $\begin{array}{l}2,90 \\
2,90\end{array}$ & $\begin{array}{l}0,97 \\
1,20\end{array}$ & 0,44 & 0,6620 \\
\hline$T A$ & 14 & $\begin{array}{l}B \\
E\end{array}$ & $\begin{array}{l}0,05 \\
0,03\end{array}$ & $\begin{array}{l}0,13 \\
0,10\end{array}$ & $\begin{array}{l}0,09 \\
0,06\end{array}$ & $\begin{array}{l}0,02 \\
0,02\end{array}$ & $-5,73$ & 0,0001 \\
\hline$T A$ & 14 & $\begin{array}{l}\mathrm{B} \\
\mathrm{E}\end{array}$ & $\begin{array}{l}0,05 \\
0,03\end{array}$ & $\begin{array}{l}0,13 \\
0,10\end{array}$ & $\begin{array}{l}0,09 \\
0,06\end{array}$ & $\begin{array}{l}0,02 \\
0,02\end{array}$ & $-5,73$ & 0,0001 \\
\hline TF & 14 & B & $\begin{array}{l}0,19 \\
0,15\end{array}$ & $\begin{array}{l}0,37 \\
0,16\end{array}$ & $\begin{array}{l}0,25 \\
0,21\end{array}$ & $\begin{array}{l}0,04 \\
0,03\end{array}$ & $-3,33$ & 0,0053 \\
\hline TATF & 14 & $\mathrm{~B}$ & $\begin{array}{l}0,24 \\
0,16\end{array}$ & $\begin{array}{l}0,49 \\
0,56\end{array}$ & $\begin{array}{l}0,36 \\
0,30\end{array}$ & $\begin{array}{l}0,07 \\
0,10\end{array}$ & $-2,24$ & \\
\hline
\end{tabular}

$\mathrm{t}=$ Valor da estatística $\mathrm{t}-$ Student; $\mathrm{p}=$ Nivel descritivo de probabilidade estatística; $\mathrm{GP}=$ Gradiente de pico; GM = Gradiente médio; $\mathrm{AV}=$ Área valvar; $\mathrm{TA}=$ Tempo de aceleraçāo; $\mathrm{TF}=$ Tempo de fluxo.

O valor máximo obtido no gradiente de pico basal (GPB) foi de $20,00 \mathrm{mmHg}$, mínimo de $3,00 \mathrm{mmHg}$, com média de $11,26 \mathrm{mmHg}$ e desvio padrão de $4,63 \mathrm{mmHg}$.

Os valores encontrados no gradiente de pico com esforço (GPE) foram: máximo de $38,80 \mathrm{mmHg}$, mínimo de $6,60 \mathrm{mmHg}$ média de $18,68 \mathrm{mmHg}$ e desvio padrão de $9,30 \mathrm{mmHg}$.
Houve uma diferença significante ( $p=0,001)$ comparando-se os gradientes de pico basal e com esforço.

Com relaçăo ao gradiente médio basal (GMB), o valor máximo obtido foi de $9,60 \mathrm{mmHg}$, mínimo de 1,40 $\mathrm{mmHg}$, com média de $5,12 \mathrm{mmHg}$, e desvio padrão de $2,14 \mathrm{mmHg}$. No gradiente médio de esforço (GME), os valores encontrados foram: máximo de $16,40 \mathrm{mmHg}$, 
POMERANTZEFF, P. M. A.; AZEVEDO, J. G.; RATTI, M.; MORAES, A. V.; AMATO, M.; GRINBERG, M.; MONTEIRO, A. C.; PUIG, L. B.; STOLF, N. A. G.; VERGINELLI, G.; JATENE, A. D. - Plástica da valva mitral em pacientes consecutivos. Como é a evolução tardia?: avaliaçāo clínica e ecocardiográfica. Rev. Bras. Cir. Cardiovasc., 6(2): 63-79, 1992.

mínimo de 3,00 , média de $8,99 \mathrm{mmHg}$ e desvio padrăo de $3,81 \mathrm{mmHg}$.

Encontra-se uma diferença significativa ( $p=$ 0,0081 ) entre os gradientes médio basal e de esforço.

$\mathrm{Na}$ área valvar em condição basal, encontramos valor máximo de $4,67 \mathrm{~cm}^{2}$, mínimo de $1,05 \mathrm{~cm}^{2}$, média de $2,90 \mathrm{~cm}^{2}$ e desvio padrão de $0,97 \mathrm{~cm}^{2}$. Os valores encontrados na área valvar com esforço foram: máximo de 4,50 , mínimo de $0,83 \mathrm{~cm}^{2}$, média de $2,90 \mathrm{~cm}^{2}$ desvio padrão de $2,00 \mathrm{~cm}^{2}$.

Comparando-se a área valvar em condições basal e com esforço não encontramos diferença significativa $(p=0,6620)$.

Não houve alteração do grau de regurgitação mitral quando o exame foi realizado com esforço, em relação à condição basal.

Cinco pacientes não apresentaram regurgitação mitral, dez pacientes apresentaram regurgitação mitral discreta e um mostrou regurgitação mitral moderada.

Tempo de aceleração em condição basal (TAB) teve seu valor máximo de $0,13 \mathrm{~s}$, mínimo de $0,05 \mathrm{~s}$, média de $0,06 \mathrm{~s}$ e desvio padrão de $0,02 \mathrm{~s}$.

Houve uma diferença significativa $(p=0,0001)$ entre os tempos de aceleração basal e com esforço.

Em condição basal, o valor máximo do tempo de fluxo (TFB) foi de $0,37 \mathrm{~s}$, mínimo de $0,19 \mathrm{~s}$, média de $0,25 \mathrm{~s}$ e desvio padrão de $0,04 \mathrm{~s}$. O valor máximo do tempo de fluxo com esforço (TFE) foi de $0,26 \mathrm{~s}$, mínimo de $0,15 \mathrm{~s}$, média de $0,21 \mathrm{~s}$ e desvio padrão de $0,03 \mathrm{~s}$.

Houve diferença significativa ( $p=0,0053$ ) entre os dois parâmetros.

\section{DISCUSSÃO}

Apesar dos esforços da Engenharia Biomédica em produzir próteses mecânicas cada vez mais perfeitas ${ }^{24}$ estas ainda não são ideiais e a durabilidade das biopróteses continua sendo um desafio para os que trabalham com a preservação dos tecidos biológicos ${ }^{21}, 93,95$. A análise tardia tem demonstrado que apesar do funcionamento hidráulico cada vez mais sofisticado das próteses mecânicas, o tromboenbolismo ainda é um problema real, requerendo a anticoagulação do paciente, terapêutica que não é fácil de ser controlada em populaçōes mais carentes. Em pacientes jovens o uso de bioprótese se defronta com o problema da calcificação precoce, e a anticoagulação não é tão simples nesta faixa etária ${ }^{5}$. 8. $14,56,58,92,108$
Com a reconstrução valvar, que preserva o tecido do próprio paciente, o risco de tromboembolismo está minimizado. A controvérsia substituição versus plástica em pacientes mitrais selecionados existe há muitos anos ${ }^{13,91,106}$ e demonstra o mérito relativo de ambos os procedimentos ${ }^{84}$. Sem dúvida a reconstrução deve ser o método escolhido quando a suficiência valvar mitral pode ser obtida sem causar estenose.

Evidentemente a reconstrução de valva mitral requer aprendizado e alguns autores têm relatado resultados não muito satisfatórios no início da experiência ${ }^{6}$. 50 .

O estudo na anatomia da valva mitral, para a avaliação das deformidades no ato operatório é realmente fundamental na obtenção de bons resultados. Além disSo, CARPENTIER et alii ${ }^{18}$ salientam que, para haver uma boa reprodutividade das técnicas, estas devem ser simples, para que a previsibilidade de bons resultados seja possivel.

CARPENTIER et alii ${ }^{17-19}$ dividiram as lesões em tipo I quando há uma movimentação normal das cúspides, porém com dilatação do anel ou perfuração de cúspide; tipo II quando existe prolapso da cúspide, devido a alongamento de cordas, ruptura de cordas ou ainda alongamento de músculo papilar ou sua ruptura e tipo III quando existe uma restrição da movimentação das cúspides, com fusão comissural, espessamento das cúspides, fusão ou espessamento das cordas.

Acreditamos que o importante além do reconhecimento das lesões, que muitas vezes estão associadas, especialmente quando se trata de febre reumática, é a sua correção através de técnica adequada, totalmente dominada pelo ciurgião.

Em todos os nossos pacientes, havia dilatação do anel posterior, e a escolha da anuloplastia adequada foi fator importante para o bom resultado; a técnica auxiliar de encurtamento de cordas proporcionou uma boa linha de coaptação das cúspides.

Devemos salientar que o número de pacientes que pode ter sua valva mitral recuperada é pequeno quando comparado ao número total de pacientes com indicação cirúrgica, isto porque, na maioria das vezes, a deformidade da valva é de tal monta que qualquer técnica de recuperação seria difícil. Este fato leva alguns autores a indagarem sobre a possibilidade de indicações mais precoces em Serviços onde a técnica da plástica da mitral está perfeitamente dominada.

A evolução clínica observada nos pacientes quanto à tolerância à atividade física foi bastante satisfatória. Como relatado nos resultados, $\mathbf{8 7 , 1 7 \%}$ dos pacientes evoluiram para classe funcional I, $7,69 \%$ para classe funcional II e 5,28\% para classe funcional III. Os dois pacientes que evoluiram para classe funcional III foram 
POMERANTZEFF, P. M. A.; AZEVEDO, J. G.; RATTI, M.; MORAES, A. V.; AMATO, M.; GRINBERG, M.; MONTEIRO, A. C.; PUIG, L. B.; STOLF, N. A. G.; VERGINELLI, G.; JATENE, A. D. - Plástica da valva mitral em pacientes consecutivos. Como é a evoluçấo tardia?: avaliação clínica e ecocardiográfica. Rev. Bras. Cir. Cardiovasc., 6(2): 63-79, 1992.

os de número 3 e 12 . O paciente de número $3 \mathrm{com}$ diagnóstico de dupla lesão mitral reumática evoluiu com insuficiência cardíaca, com evolução da lesão mitral para insuficiência além de apresentar lesão aórtica. Foi submetida a plástica de valva aórtica e substituição da valva mitral com 54 meses de evolução em outro Serviço. O paciente de número 12 com diagnóstico de dupla lesão mitral reumática foi reoperado aos 19 meses de evolução, sendo possível realizar nova plástica da mitral, atualmente encontrando-se em classe funcional I.

Estes dados são comparáveis aos da literatura ${ }^{22}$. 32, 60 . LESSANA et alii ${ }^{66}$ relatam $80,76 \%$ de pacientes em classe funcional I no pós-operatório tardio, analisando 130 pacientes operados de janeiro de 1975 a janeiro de 1982

CARPENTIER et alii ${ }^{18}$ analisando a evoluçāp de dez anos em 551 pacientes operados de janeiro de 1969 a janeiro de 1978 relatam $76 \%$ dos pacientes em classe funciona $\mathrm{I}$.

ANTUNES et alii ${ }^{7}$ em trabalho em que analisaram 241 pacientes reumáticos submetidos a plástica mitral relatam $85 \%$ dos pacientes em classe funcional I com evolução de 576 pacientes/ano (média de 2,64 $\pm 1,32$ ano).

YACOUB et alii ${ }^{105}$ compararam os resultados da plástica da mitral e substituição mitral em pacientes portadores de degeneração mixomatosa, sendo 86 submetidos a plástica e 46 a substituição valvar. A plástica utilizada foi a anuloplastia tipo Merendino sem utilização de anel protético. Com evolução média de 38 meses, $94 \%$ dos pacientes submetidos a plástica valvar mitral estavam em classe funcional I, contra $77 \%$ dos pacientes submetidos a troca valvar, com evolução média de 96 meses.

A mortalidade operatória dos nossos pacientes submetidos a plástica mitral se compara aos dados da literatura que está entre 0 e $6,3 \% 7,8,41,74,79-81$. A mortalidade relatada nas substituiçōes valvares geralmene é maior ${ }^{14}$. 75. 83. Os nossos resultados em relaçāo às estimativas de funçăo de sobrevida dos pacientes, de sobrevida da plástica livre de reoperação e da função livre de eventos morte e reoperação se assemelham aos resultados de outros autores. COSGROVE et alii ${ }^{27}$ relatam sobrevida actuarial dos pacientes de $90,6 \%$ em dois anos, e ANTUNES et alii ${ }^{6}, 90 \%$ em quatro anos e meio. Estes últimos relatam $82 \%$ de estimativa de sobrevida da plástica livre de reoperações. SHORE et alii ${ }^{94}$ relatam $89 \%$ de sobrevida em três anos em pacientes com idade média de 55 anos. REED et alii ${ }^{88}$ descreveram os seus resultados em 196 pacientes operados entre janeiro de 1961 e janeiro de 1968 relatando mortalidade hospitalar de $4,5 \%$ e tardia de $8,7 \%$, com $97 \%$ dos pacientes livres de tromboembolismo em 17 anos de evolução.
Têm sido descritas taxas linearizadas baixas ${ }^{18,26}$ para fenômenos tromboembólicos e endocardite bacteriana nas reconstruçōes mitrais, dados estes que coincidem com os que nós encontramos.

Com relação ao evento reoperação, CARPENTIER et alii ${ }^{18}$ relatam taxa de $0,7 \%$ por paciente/ano e ANTUNES et alii $^{7}$ relatam índice de reoperação de $7,33 \%$ por paciente/ano em pacientes reumáticos em idade igual ou inferior a 12 anos e de $3,29 \%$ em pacientes com idade maior de 12 anos.

COSGROVE et alii ${ }^{27}$ relatam $2,5 \%$ de reoperações por paciente/ano tendo na sua casuística $80 \%$ dos pacientes com lesōes degenerativas.

A nossa taxa de reoperaçōes foi de 1,6\% por paciente/ano, sendo que em nossa casuística houve predominância de pacientes portadores de febre reumática; os dois pacientes reoperados apresentavam esta etiologia. Outros relatam taxas ainda maiores de reoperaçăo em pacientes reumáticos ${ }^{33,98}$.

Como foi relatado nos nossos resultados 19 pacientes continuaram com sopro sistólico + , seis com sopro de ++ e um com sopro de +++ . YACOUB et alii ${ }^{106}$ relatam desaparecimento de sopro em $45 \%$ dos seus pacientes, permanecendo sopro de $+/++$ em $26,7 \%$, e em dois pacientes, correspondendo a $2,3 \%$ sopro de $++1+++$, o que dá um total de $74 \%$. A nossa taxa de pacientes que permaneceu com sopro sistólico foi de $66,66 \%$. O paciente de número 6 que apresenta sopro sistólico $++1+++$ encontra-se em classe funcional I.

Vários autores $59,70,77,101,102$ têm utilizado a ecocardiografia e a ecodopplercardiografia para avaliação préoperatória em pacientes com lesōes valvares, e para sua posterior avaliação tardia, dispensando desta maneira métodos invasivos.

Além disso, existe um crescente interesse no uso da ecocardiografia para estudo da funçāo ventricular esquerda, já que as suas dimensōes podem ser acuradamente medidas pela ecocardiografia unidimensional, através da distância entre o lado esquerdo do septo interventricular e o endocárdio da parede posterior do ventrículo esquerdo ${ }^{35,36}$. Na literatura encontramos estudos que mostram uma boa correlação entre as dimensões medidas pela ecocardiografia e o menor eixo do ventrículo esquerdo medido pela angiografia ${ }^{37,}{ }^{42}$. A redução proporcional nos diâmetros entre o final da diástole e o final da sístole correlaciona-se diretamente com a fração de ejeção ${ }^{66,67}$.

Alguns trabalhos 3, 9,41 mostram a regressāo das cavidades esquerdas após correçāo cirúrgica de lesōes mitrais. SHERRID et alii ${ }^{93}$ relatam diminuição significativa do átrio esquerdo em pacientes que tiveram evolução clínica satisfatória após cirurgia de valva mitral e manu- 
POMERANTZEFF, P. M. A.; AZEVEDO, J. G.; RATTI, M.; MORAES, A. V.; AMATO, M.; GRINBERG, M.; MONTEIRO, A. C.; PUIG, L. B.; STOLF, N. A. G.; VERGINELLI, G.; JATENE, A. D. - Plástica da valva mitral em pacientes consecutivos. Como é a evolução tardia?: avaliação clínica e ecocardiográfica. Rev. Bras. Cir. Cardiovasc., 6(2): 63-79, 1992.

tenção da dimensão do mesmo nos casos que tiveram má evolução.

Os nossos resultados demonstrando a regresão significativa da dimensão diastólica do ventrículo esquerdo além de significativa redução do tamanho do átrio esquerdo corroboram estes dados.

Analisando, isoladamente o paciente de número 12 que necessitou reoperação, notamos que, no período de pós-operatório, os diâmetros sistólico e diastólico do ventrículo esquerdo aumentaram, assim como o diâmetro do átrio esquerdo.

Deste modo, os dados relativos às dimensōes das cavidades mostraram que 0 acompanhamento ecocardiográfico modo $\mathrm{M}$ foi importante para demonstrar a regressão do tamanho do ventrículo esquerdo e do átrio esquerdo.

Em nossos pacientes o $\mathrm{D} \%$ médio pré-operatório foi de $35,76 \%$ com desvio padrão de 6,73 e pós-operatório de $33,73 \%$ com desvio padrão de $6,43 \%$, não havendo diferença significativa $(p=0,1440)$, porém, com valores um pouco inferiores no pós-operatório. Este dado, adquirido na última avaliação dos pacientes, indiretamente reflete a ausência de regurgitação mitral significativa, pois a presença desta faz com que o $\mathrm{D} \%$ esteja aumentado, em função da pós-carga diminuída ${ }^{44,100}$.

ADEBO \& ROSS ${ }^{3}$ acreditam que a dimensão diastólica final do ventrículo esquerdo é um ótimo índice da função ventricular esquerda pós-plástica de valva mitral nos casos em que existe movimentação anormal do septo interventricular, fato já descrito na literatura ${ }^{63}$. Esta movimentação anormal não foi notada em nossos pacientes, mas a redução da dimensão diastólica foi notória.

Nos 16 pacientes submetidos a avaliação ecodopplercardiográfica os gradientes de pico e médio através da valva mitral foram respectivamente de $11,26 \mathrm{mmHg}$ com desvio padrão de 4,63 e $5,12 \mathrm{mmHg}$ com desvio padrão de 2,14. Por sua vez a área valvar mitral média derivada do Doppler nestes pacientes foi de $2,9 \mathrm{~cm}^{2}$ com desvio padrão de 0,97 , parâmetros semelhantes aos encontrados na literatura em pacientes submetidos a plástica mitral ${ }^{61}$.

O nosso grupo de pacientes quando submetido a esforço apresentou elevaçāo significativa dos gradientes de pico e médio através da mitral, porém sem alteração signinificativa de $(p=0,6620)$ da área valvar, sugerindo, portanto, que esta área não sofre grandes alterações a despeito da elevação dos gradientes transvalvares causados pelo esforço e aumento da freqüência cardía- ca, porque, provavelmente, não esteja representando uma área anatômica, e sim hemodinâmica ${ }^{52,53}$,

É importante salientarmos que, pelo estudo ecodopplercardiográfico, dois pacientes apresentam estenose mitral moderada em um paciente é importante em outro, estando os mesmos respectivamente com 79 e 78 meses de evolução, e em classe funcional l.

Estudos ${ }^{104,} 105$ realizados em pacientes portadores de prótese demonstraram gradientes maiores do que os encontrados em pacientes com plástica da mitral, quando avaliados pela ecodopplercardiografia.

O mapeamento de fluxo a cores e com Doppler pulsado nos 16 pacientes estudados revelou regurgitação mitral moderada em em paciente, discreta em dez pacientes e nenhuma regurgitação em cinco. Este mesmo grupo quando submetido ao teste de esforço não apresentou aumento do refluxo mitral em nenhum paciente.

O tempo de aceleração (TA) e o tempo e fluxo (TF) na artéria pulmonar foram estudados com o objetivo de avaliar as repercussōes hemodinâmicas em território pulmonar.

Comparando-se os nossos resultados com os valores normais indicados na literatura ${ }^{52,84}$ vemos que os pacientes não apresentam hipertensão importante.

A plástica valvar mitral, apesar de requerer aprendizado e experiência e ter sua aplicabilidade reduzida pela deformação acentuada da valva em muitos casos, está sendo cada vez mais utilizada devido aos seus bons resultados, com baixos índices de mortalidade e morbidade ${ }^{33,44}$.

Embora as lesôes degenerativas tenham a sua meIhor indicação ${ }^{2,20,34}$, a plástica poderá ser utilizada criteriosamente em pacientes com outras doenças ${ }^{23,38,41 .}$

Outra consideração que deve ser feita é que a ruptura ventricular ${ }^{25,74}$, complicação muito grave após troca valvar mitral, não tem sido relatada em pacientes submetidos a plástica da mitral.

A obstrução da via de saída do VE relatada por alguns autores ${ }^{40,63}$ não foi observada em nossos pacientes, talvez porque foram utilizadas várias técnicas de anuloplastia, tentando adequar a melhor para cada caso.

Acreditamos que o estado atual da plástica valvar permite sua indicação antes que lesōes miocárdicas irreversíveis ocorram. 
POMERANTZEFF, P. M. A.; AZEVEDO, J. G.; RATTI, M.; MORAES, A. V.; AMATO, M.; GRINBERG, M.; MONTEIRO, A. C.; PUIG, L. B.; STOLF, N. A. G.; VERGINELLI, G.; JATENE, A. D. - Plástica da valva mitral em pacientes consecutivos. Como é a evoluçāo tardia?: avaliaçāo clínica e ecocardiográfica. Rev. Bras. Cir. Cardiovasc., 6(2): 63-79, 1992.

RBCCV 44205-134

POMERANTZEFF, P. M. A.; AZEVEDO, J. G.; RATTI, M.; MORAES, A. V.; AMATO, M.; GRINBERG, M.; MONTEIRO, A. C.; PUIG, L. B.; STOLF, N. A. G.; VERGINELLI, G.; JATENE, A. D. - Mitral valve reconstruction: long-term follow-up. Rev. Bras. Cir. Cardiovasc., 6(2): 63-79, 1991.

ABSTRACT: Thirty-nine patientes with mitral insufficiency or mitral stenosis and incompetence submitted to valvular repair were evaluated in the late postoperative period. Mean age of the patients was 30.5 years, with standard deviation of 17.2 years. Twenty-four patients were females $(61.5 \%)$ and $15(38.5 \%)$ males. Twenty-one Carpentier ring annuloplasties, 12 repair with a posterior sling, five Merendino type annuloplasties and one Kay type annuloplasty were performed. Twenty-three $(58.97 \%)$ patients presented symptons related to rheumatic fever disease, $12(30.76 \%)$ had no definite etiology and four $(10.25 \%)$ presented mucoid degeneration occurred in this series. Evolution time was 1497 months/standard (meam 38.39 months and Standard deviation of 16.08 months). In the late postoperative period $34(87.74 \%)$ patients were in NYHA functional class I. Two late deaths $(5.12 \%)$ occurred, and two $(5.12 \%)$ patients were reoperated on. Linearilized rates of the reoperation and thromboembolism events were $1.6 \%$ and $0.8 \%$ per patient/year, respectively. Estimated survival rate was $94.87 \%$. Echocardiographic evaluation of the pre and postoperative values demonstrated significant decrease of the left ventricular diastolic diameter $(p=0.0001)$, of the left ventricular systolic diameter $(p=0.0001)$ of the left venticular systolic diameter $(p=0.0001)$ and of the left atrial diameter ( $p=0.0001$ ). The postoperative Doppler echocardiograpfic studies demonstrated absence of valvular area changes at effort. It was possible to conclude that patients submitted to mitral valve repair benefited from higher survival rates and satisfactory clinical evolution.

DESCRIPTORS: heart valves, mitral, surgery.

\section{REFERÊNCIAS BIBLIOGRÁFICAS}

1 ABBASSI, A. S.; ALLEN, M. W.; DECRISFOFARD, D.; UN GAR, I. - Detection and estimation ot the degree of mitral regurgitation by rangegated pulsed Doppler echocardiography. Circulation, 61: 143-147, 1980.

2 ACAR, J.; CARAMANIAN, M.; PERRAULT, M.; LUXEREAU, P.; ARNAUD, J. C. - Les insuffisances mitrales par ruptures de cordages d'origine dégenérative. Arch. Mal. Coeur, 61: 1724-1737, 1968.

3 ADEBO, O. A. \& ROSS, T. K. - Conservative sugery for mitral valve disease: clinical and echocardiographic analyis of results. Thorax, 38: 565-571, 1983.

4 ANGELL, W. W.; OURY, J. H.; SHAH, P. - A comparison of replacement and reconstriction in patients with mitral regurgitation. J. Thorac. cardiovasc. Surg., 93: 665-674, 1987.

5 ANGER, L. E.; PATE, J. W.; LAWYER, G.; FITCH, C. W.; SHERMAN, P. H. - Prosthetic mitral valve replacement in children. J. Pediatr., 70: 577-585, 1967.

6 ANTUNES, M. J.; COLSEN, P. R.; KINSLEY, R. H. Mitral valvuloplasty: a learning curve. Circulation, 68 (Supl. 2): 1179-1175, 1983.

7 ANTUNES, M. J.; MAGALHĀES, M. P.; COLSEN, P. R.; KINSLEY, R. H. - Valvuloplasty for rheumatic mitral valve disease: a surgical challenge. J. Thorac. Cardiovasc. Surg., 94: 44-56, 1987.
8 ARMISTEAD, S. H.; MACFARLAND, R.; LANE, I.; PANETH, M. - Mitral valve surgery in infants and children. J. Cardiovasc. Surg., 24: 144-149, 1983.

9 BAYLEY, C. P.; JAMISON, W. L.;BAKST, A. E.; BOLTON, H. E.; NICHOLS, H. T.; GEMEINHARDT, W. - The surgical correction of mitral insufficiency by the use of pericardial grafts. J. Thorac. Surg., 28: 551-603, 1954.

10 BERNARD, R. - Fundamentals of Biostatistics. Boston, PWS Publishers, 2. ed. 1986, p. 140-246.

11 BLACKSTONE, E. H. \& KIRKLIN, J. W. - Death and other time related events after valve replacement. Circulation, 72: 753-767, 1985

12 BODMAR, E.; HABERMAN, S., WAIN, W. H. - Compative method for actuarial analysis of cardiac valve replacements. Br. Heart J.,42: 541-552, 1979.

14 BORKON, A. M.; SOULE, L.; REITZ, B. A.; GOTT, V. L.; GARDNER, T. J. - Five year follow-up after valve replacement with the St. Jude Medical valve in infants. and children. Circulation, 74 (Supl. 1): 110-115, 1986.

15 BRAUNWALD, E. - História. In: Tratado de medicina Cardiovascular 2. ed. São Paulo. Roca, 1987. Cap. 1, p. 3-13.

16 CARPENTIER, A. - La valvuloplastie reconstitutive: une nouvelle tehnique de valvuloplastie mitrale. Presse Med., 77: 251-253, 1969. 
POMERANTZEFF, P. M. A.; AZEVEDO, J. G.; RATTI, M.; MORAES, A. V.; AMATO, M.; GRINBERG, M.; MONTEIRO, A. C.; PUIG, L. B.; STOLF, N. A. G.; VERGINELLI, G.; JATENE, A. D. - Plástica da valva mitral em pacientes consecutivos. Como é a evolução tardia?: avaliação clínica e ecocardiográfica. Rev. Bras. Cir. Cardiovasc., 6(2): 63-79, 1992.

17. CARPENTIER, A.; BRANCHINI, B.; COURT, J. C.; ASFAOU, E.; VILLANI, M.; DELOCHE, A.; RELLAND, J.; D'ALLAINES, C. L.; BLONDEAU, Ph.; PIWNICA, A.; PERENZAN, L.; BROM, G. - Congenital malformations of the mitral valve in children: pathology and surgical treatment. J. Thorac. Cardiovasc. Surg., 72: 865-866, 1976.

18 CARPENTIER, A.; CHAUVAUD, S.; FABIANI, J. N.; DELOCHE, A.; RELLAND, J.; LESSANA, A.; D'ALLAINES, Cl.; BLONDEAU, Ph.; PIWNICA, A.; DUBOST, Ch. - Reconctructive surgery of mitral valve incompetence: ten-year appraisal. J. Thorac. Cardiovasc. Surg., 79: 338-348, 1980.

19 CARPENTIER, A.; DELOCHE, A.; DAUPATAIN, J.; SOYER, R.; BLONDEAU, P.; PIWNICA, A.; DUBOST, Ch.; McGOON, D. C. - A new reconstructive operation for correction of mitral and tricuspid insufficiency. $J$. Thorac. Cardiovasc. Surg., 61: 1-13, 1971.

CARPENTIER, A.; RELLAND, J.; DELOCHE, A. P.; PIWNICA, A.; CHAUVAUD, S.; DUBOST, C. - Conservative management of the prolapsed mitral valve. Ann. Thorac. Surg., 26: 294-302, 1978.

CHAFFIN, J. S. - Mitral valve replacement: a nine-year follow-up of risks and survivals. Ann. Thorac. Surg., 27: 312-319, 1978.

22 CHARLESWORTH, D. C.; WEISEL, R. D.; BAIRD, R. J.; SWLLY, H. E. - Assessment of mitral and tricuspid competence after valvuloplasty. Ann. Thorac. Surg., 35: 105-107, 1983.

CHAUVAND, S.; PERIER, P.; TOUATI, G.; RELLAND, J.; KARA, S. M.; BENOMAR, M.; CARPENTIER, A. - Long-term results of valve repair in children with acquired mitral valve incompetence. Circulation, $\mathbf{7 4}$ (Supl. 1): 104-109, 1986.

24 CHAUX, A.; CZER, L. S. C.; MATLOFF, J. M.; DEROBERTIS, M. A.; STEWART, M. E.; BATEMAN, T. M. KASS, R. M.; LEE, M. E.; GRAY, R. J. - The St Jude Medical bileaflet valve prosthesis. J. Thorac. Cardiovasc. Surg., 88: 706-717, 1984

25 COBBS Jr., B. W.; HATCHER Jr., C. R.; CRAVER, J. M.; JONES, E. L.; SWELL, C. W. - Transverse midventricular disruption after mitral valve replacement. Am. Heart. J., 99: 33-49, 1980.

26 COSGROVE, D. M. - Mitral valve prolapse: surgical management. In: BOUPOLAS, H.; WOOLEY, C. F. Mitral valve prolapse and the mitral valve prolapse syndrome. Mount Kisco, Futura Publishing Co, 1988, cap. 19 , p. 345-374.

27 COSGROVE, D. M.; CHAVEZ, A. M.; LYTLE, B. W.; GILL, C. C.; STEWART, R. W.; TAYLOR, P. C.; GOORMASTIC, M.; BORSH, J. A.; LOOP, F. D. - Results of mitral valve reconstruction. Circulation, 74 (Supl. 1): 82-87, 1986.
28 DAVACHI, F.; MOLLER, J. H.; EDWARDS, J. E. - Diseases of the mitral valve in infancy. Circulation, 53: 565-579, 1971.

29 DAVID, T. E.; KOMEDA, M.; POLLICK, C.; BURNS, R. J. - Mitral valve annuloplasty: the effect of the type on left ventricular function. Ann. Thorac. Surg., 47: 524-528, 1989.

30 DAVILA, J. C.; MATTSON Jr., W. W.; O'NEILL, T. J. E.; GLOVER, R. P. - A method for the surgical correction of mitral insufficiency. Surg. Ginecol. Obstet., 98: 407-412, 1954

31 DEL CASTILHO, J. M.; MORAES SOBRINHO, O. H.; VASCONCELOS, P. F.; RESENDE, C.A.; ARIÊ, S.; GALIANO, N.; ARMELIN, E. - Aspectos ecocardiográficos das próteses de dura-máter. Arq. Bras. Cardiol., 37: 181-187, 1981.

32 DUBOST, C. - Evaluation of surgery for mitral valve disease. Am. Heart J., 82: 143-148, 1971.

33 DURAN, C. G.; REVUELTA, J. M.; GAITE, L. I.; ALONSO, C.; FLEITAS, M. G. - Stability of mitral reconstructivesurgery at 10-12 years for predominantly rheumatic valvular disease. Circulation. 78: 91-96, 1988.

34 ELLIS Jr, F. H.; FRYE, R. L.; McGOON, D. C. - Results of reconstrutive operations for mitral insufficiency due to ruptured chordae tendineae. Surgery, 59: 165-172, 1966.

35 FEIGENBAUN, H.; POPP, R. L. ; WOLFF, S. B.; TROY, B. L.; POMBO, J. F.; HAINE, C. L.; DODGE, H.T. Ultrasound measurements of the left ventricle. Arch. Intern. Med., 129: 461-467, 1972.

36 FEIGENBAUM, H.; WOLFE, S. B.; POPP, R. L.; HAINE, C. L.; DOUGE, H. T. - Correlation of ultrasound with angiocardiography in measuring left ventricular diastolic volume. Am. J. Cardiol.. 23: 111-114, 1969.

37 FORTUIN, N. J.; HOOD Jr, W. P.; SHERMAN, M. E.; CRAISE, E. - Determination of left ventricular volumes by ultrasound. Circulation, 54: 575-584, 1971.

38 FRATER, R. W. M. - Aggressive attempts at mitral valve plasty: is there a mice? In: BONDNAR, E. (ed.) Surgery for heart valve disease. 1990, p. 147-153.

39 FRATER, R. W. M.; GABBAY, S.; SHOIRE, D.; FACTOR, S.; STROM, J. - Reproducible replacement of elongated or ruptured mitral valve chordar. Ann. Thorac. Surg., 35: 14-20, 1983.

40 GALLER, M.; KRONZON, I.; SLATER, J.; LIGHTY Jr., F. W.; POLITZER, F.; COLVIN, S.; SOEBCER, F. Long-term follow-up after mitral valve reconstruction: incidence of pos-toperative left ventricular outflow obstruction. Circulation, 74(Supl. 1): 99-103, 1986.

41 GallowAY, A. C.; COLVIN, S. B.; BAUMANN, F. G.; GROSSI, E. A.; RIBAKOVE, G. H.; HARTY, S.; SPEN- 
POMERANTZEFF, P. M. A.; AZEVEDO, J. G.; RATtI, M.; MORAES, A. V.; AMATO, M.; GRINBERG, M.; MONTEIRO, A. C.; PUIG, L. B.; STOLF, N. A. G.; VERGINELLI, G.; JATENE, A. D. - Plástica da valva mitral em pacientes consecutivos. Como é a evolução tardia?: avaliação clínica e ecocardiográfica. Rev. Bras. Cir. Cardiovasc., 6(2): 63-79, 1992.

CER, F. C. - A comparison of mitral valve reconstruction with mitral valve replacement: intermediate term results. Ann. Thorac. Surg., 47: 655-622, 1989.

GIBSON, D. G. - Estimation of left ventricular size by echocardiography. Br. Heart. J., 35: 128-134, 1973.

43 GOLDMAN, M. E.; MINDICH, B. P.; TEICHOOLZ, L. E.; BURGESS, N.; STAVILLE, K.; FUTER, V. - Intraoperative contrast echocardiography to evaluate mitral valve operations. J. Am. Coll. Cardiol., 4: 1035-1040, 1984.

44 GOLDMAN, M. E.; MORA, F.; GUARINO, T.; FUSTER, V.; MINDICH, B. P. - Mitral valvuloplasty is superior to valve replacement for preservation of left ventricular function; an intraoperative two-dimensional echocardiographic study. J. Am. Coll. Cardiol., 10: 568-575, 1987.

GREGORI Jr., F.; TAKEDA, R. T.; SILVA, S.S.; FAÇANHA, L. A.; RIBEIRO, J. A.; AQUINO, W. F.; SHIGUERU, S.; GOIS, L. E.; SIQUEIRA, J. E.; KRELING, P. A.; GOULART, M. P.; FABIANO, M.; GRULAN, A. I.; MIGUITA, L. C.; CANESIN, O. - Sete anos de experiência com plastia de valva mitral. Arq. Bras. Cardiol., 47: 269-274, 1986.

GROSMAN, W. - Profiles in valvular heart disease In: Cardiac. catheterization and angiopgraphy. 3 ed. 1986 , p. $359-381$

47 GRUNKEMEIER, G. L. - Statistical analysis of prothetic valve series. In: RABAGO, G. L. \& COOLEY, D. A. Heart valve replacement. 1987, p. 2-26.

48 GRUNKEMEIER, G. L. \& STARR, A. - Actuarial analysis of surgical results: ratinale and method. Ann. Thorac. Surg., 24: 404-408, 1977.

GUVENDIK, L.; COBANOGLU, A.; DAVIS, N. R.; FESSLER, C. L.; STARR, A. - Valve replacement in children under 14 years of age: a review of 22 years of experience. Eur. J. Cardio-Thorac. Surg., 3: 229-234, 1989.

50 HANANIA, G.; DELOCHE, A.; MARCANTONI, J. P.; GERBAUX, A. - Resultats à moyen terme de l'annuloplastie mitrale reconstrutive de Carpentier. Arch. Mal. Coeur., 66: 1297-1309, 1973.

51 HANSEN, D. E.; CAHILL, P. D.; DERBY, G. C.; MILLER, D. C. - Relative contributions of the anterior and posterior mitral chordae tendineae to canine global left ventricular systolic function. J. Thorac. Cardiovasc. Surg., 93: $45-55,1987$

HARKEN, D. H.; BLACK, H.; ELLIS, L. B.; DEXTER, L. - The surgical correction of mitral insufficiency. $J$. Thorac. Surg., 28: 604-627, 1954.

HATLE, L. \& ANGELSEN, B. - Dopplerultrasound in cardiology. Philadelphia, LEA \& FEBIGER, 1985, p. 97-292.
54 HATLE, L.; ANGELSEN, B.; TROMSDAL, A. - Noninvasive assessment of atrioventricular pressure half-time Doppler ultrasound. Circulation, 60: 1096-1104, 1979.

55 HESSEL, E. A.; KENNEDY, J. W.; MERENDINO, K. A. - A reappraisal of nonprosthetic reconstructive surgery of mitral regurgitation based on an analysis of early and late results. J. Thorac. Cardiovasc. Surg., 52: 193-206, 1966.

56 SIHARA, T.; FERRANS, W. J.; JONES, M.; CABIN, H. S.; ROBERTS, W. C. - Calcific deposits developing in a bovine pericardial bioprosthetic valve 3 days after implantation. Circulation, 63: 718-728, 1981.

57 HAHN, D. R.; STERN, A. M.; SIGMANN, J. M.; KIRSH, M. M. - Longterm resuls of mitral insufficiency in children. J. Thorac. Cardiovasc. Surg., 53: 1-6, 1967.

58 KARASIK, A.; HALPERIN, Z.; LEWIS, B. S.; GEFT, I. L.; BORMAN, J. B.; GOTSMAN, M. S. - Echocardiografic left ventricular function in aortic and mitral incompetence and the effect of valve replacement. IsraelJ. Med. Sci., 13: 1171-1181, 1977.

59 KAY, G. L.; KAY, J. H.; ZUBIATE, P.; YOKOYAMA, T.; MENDEZ, M. - Mitral valve repair for mitral regurgitation secondary to coronary artery disease. Circulation, 74 (Supl. 1): 88-98, 1986.

60 KENNEDY, J. W.; DOCES, J. G.; STEWART, D. K. Left ventricular function before and following surgical treatment of mitral valve disease. Am. Heart J., 97: 592-598, 1979.

61 KENNY, J.; COHN, L.; CHEMIN, R.; COLLINS, J. J.; PLAPPERT, M.; SUTTON, M. G. St. J. - Doppler echocardiographic evaluation of ring mitral valvuloplasty for pure mitral regurgitation. Am. J. Cardiol., 59: $341-345,1987$.

62 KREINDEL, M. S.; SCHIFNONE, W. A.; LEVER, H. M.; COSGROVE, D. - Systolic anterior motion of the mitral valve after Carpentier ring valvuloplasty for mitral valve prolapse. $\quad A m$. J. Cardiol., 57: 408-412, 1986.

63 LAM, J. H. C.; RANGANATHAM, N.; WIGLE, E. D.; SILVER, M. D. - Morphology of the human mitral valve. Circulation, 51: 449-458, 1970.

64 LESSANA, A.; ESCORSIN, M.; ROMANO, M.; ADES, F.; VERGON, W.; RENZONI, D.; MENOZZO, C. C.; MONDUCCI, I. - Transposition of posterior leaflet for treatment of ruptured main chordae of the anterior mitral leaflet. J. Thorac. Cardiovasc. Surg., 89: 804-806, 1985.

65 LESSANA, A.; VIET, T. T.; ADES, F.; KARA, S. M. AMEUR, A.; RUFFENACH, A.; UERIN, F.; HERREMAN, F.; DEGEORGES, M.; FRATER, R. W. M. Mitral reconstructive operations: a series of 130 consecutive cases. J. Thorac. Cardiovasc. Surg., 86: 553-561, 1983. 
POMERANTZEFF, P. M. A.; AZEVEDO, J. G.; RATTI, M.; MORAES, A. V.; AMATO, M.; GRINBERG, M.; MONTEIRO, A. C.; PUIG, L. B.; STOLF, N. A. G.; VERGINELLI, G.; JATENE, A. D. - Plástica da valva mitral em pacientes consecutivos. Como é a evolução tardia?: avaliação clínica e ecocardiográfica. Rev. Bras. Cir. Cardiovasc., 6(2): 63-79, 1992.

66 LEWIS, J. F.; KVO, L. C.; NELSON, J. G.; LIMACHER, M. C.; QUINONES, M. A. - Pulsed Doppler echocardiographic determination of stroke volume and cardiac output: clinical validation of two new methods using the apical window. Circulation, 70: 425-431, 1984.

67 LEWIS, R. P. \& SASNDLER, H. - Relationship between changes in left ventricular dimensions and the ejection fraction in man. Circulation, 54: 58-557, 1987.

68 LILLEHEI, C. W.; GOTT, V. L.; DEWALL, R. A.; VARCO, R. L. - Surgical correction of pure mitral insufficiency by annuloplasty under direct vision. Lancet, 77: 406-409, 1957

69 McGOON, D. C. - Repair of mitral insufficiency due to ruptured chordae tendinea. J. Thorac. Cardiovasc. Surg., 39: 357-362, 1960.

70 MYATAKE, K.; KINOSHITA, N.; NAGATA, S.; BEPPUS, S.; PARK, Y. D.; SAKAKIBARA, H.; NMURA, Y. Intracardiac flow pattern in mitral regurgitation studied with combined use of the ultrasonic pulsed Doppler technique and cross-sctional echocardiography. Am. J. Cardiol, 45: 155-162, 1980.

71 NANDA, N. C. - Atlas of color Doppler echocardiographic. Philadelphia, Lea \& Febiger, 1989, cap. 3, p. 46-110.

72 NICHOLS, H. T. - Mitral insufficiency treatment by polar crossOfusion of the mitral, annulus fibrosus. J. Thorac. Cardiovasc. Surg., 33: 102-122, 1957.

73 NILI, M.; SALOMON, J.; HALEVI, A.; SCHUCHMAN, E.; LEVY, M. J. - Left ventricular rupture after mitral valve replacement. Scand. J. Thorac. Cardiovasc. Surg., 15: 235-238, 1981.

74 OURY, J. H.; PETERSON, K. L.; FOLKERTH, T. L.; DAILY, P. O. - Mitral valve replacement versus reconstruction: an analysis of indications and results of mitral valve procedures in a consecutive sries of 80 patients. J. Thorac. Cardiovasc. Surg., 73: 825-835, 1977.

75 PENTHER, P. - Traitement chirurgical pour annuloplastie des insuffisances mitrales: résultat a long-terme. Presse Med., 77: 1885-1886, 1969.

76 PERLOFF, J. K. \& ROBERTS, W. C. - The mitral apparatus: functional anatomy of mitral regurgitation. Circulation, 56: 227-239, 1972.

77 PERRY, L. W.; MIDGLEY, F. M.; GALIOTO Jr., F. M.; SHAPIRD, S. R.; RUCKMAN, R. N.; SCOTT, L. P. - Two-dimensional echocardiographic evaluation of mitral bioprosthetic function in infants and children. Am. Heart J., 102: 1022-1028, 1981.

78 PINSON, C. W.; COBANOGLU, A.; METZDORFF, M. A. - Late surgical results for ischemic mitral regurgitation: role of regurgitation. J. Thoracic. Cardiovasc. Surg., 88 (Supl. 5, Parte 1): 663-672, 1984.
79 POMERANTZEFF, P. M. A.; AMATO, M.; STOLF, N. A G.; MARCIAL, M. B.; GRINBERG, M.; PILEGGI, F.; VERGINELLI, G.; JATENE, A. D. - Experiência com plástica de valva mitral. Arq. Bras. Cardiol., 45 (Supl. 1): 156, 1985. (Resumo).

80 POMERANTZEFF. P. M. A.; PIERACCIANI, G.; PUIG, L. B.; FONTES, R. D. - Insuficiência mitral. In: Clínica cirúrgica Alipio Correa Neto. 4. ed. São Paulo, Sarvier, 1988. Cap. 81, p. 719-731.

81 POMERANTZEFF, P. M. A.; STOLF, N. A. G.; AMATO, M.; MORETTI, M.; GRINBERG, M.; MORAES, A. V.; CAMARANO, G.; AULER Jr., J. O. C.; VERGINELLI, G.; JATENE, A. D. - Plástica de valva mitral (PVM): experiência do InCor. Arq. Bras. Cardiol., 49 (Supl. 1): 161, 1987. (Resumo).

82 POMERANTZEFF, P. M. A.; ZERBINI, E. J.; VERGINELLI, J.; JANETE, A. D. - Valve replacement in the Heart Institute, University of Sâo Paulo, Brasil. Ann. Thorac. Surg., 48: 541-544, 1989.

83 PUIG, L. B.; VERGINELLI, G.; SOSA, E. A.; ROMA, L S. L.; GARGIA, D. P.; CONCEIÇĀO, A. N.; ZERBINI, E. J.; PILEGGI, F. - Avaliação hemodinníca da valva de dura-máter mitral e tricúspide. Arq. Bras. Cardiol., 29: $297-301,1976$

84 QUINOMES. M. A. - Doppler evaluation of right sided lesions and pulmonary hypertension In: NANDA, N. C. (ed.) Dopplerechocardiography. New York, IgakuShoin, 1985. Cap. 13, p. 262-292.

85 RADFORD, M. J.; JOHNSON, R. A.; BUCKLEY, M. J.; DAGGETT, W. M.; LEINBAC, R. C.; GOLD, H. K. Survival following mitral valve replacement for mitral regurgitation due to coronary artery disease. Circulation, 60 (Supl. 1): 39-47, 1979.

86 RANGANATHAN, N.; LAM, J. H. C.; WIGLE, E. D.; SILVER, M. D. - Morphology of the human mitral valve. Circulation, 51: 459-467, 1970.

87 RAPAPORT, E. - Natural history of aortic and mitral valve disase. Am. J. Cardiol., 45: 221-227, 1975.

88 REED, G. E.; POOLEY, R. W.; MOGGIO, R. A. - Durability of measured mitral annuloplasty: seventeen-year study. J. Thorac. Cardiovasc. Surg., 79: 31-35, 1980.

89 ROBERTS, W. C. \& MORROW, A. G. - Causes of early postoperative death following cardiac valve replacement: clinical pathologic correlations in 64 patients studied at necropsy. J. Thorac. Cardiovasc. Surg., 54: 422-437, 1967.

90 SAND, M. E.; NAFTEL, D. C.; BLACKSTONE, E. H. KIRKLIN, J. W.: KARP, R. B. - A comparison of repair and replacement for mitral valve incompetence. $J$. Thorac. Cardiovasc. Surg., 94: 208-219, 1987.

91 SAS, Institute Inc.; SAS/STAT - Guide personal computers. version 6, Edition Cary, NC: SAS Institute Inc., 1987. 
POMERANTZEFF, P. M. A.; AZEVEDO, J. G.; RATTI, M.; MORAES, A. V.; AMATO, M.; GRINBERG, M.; MONTEIRO, A. C.; PUIG, L. B.; STOLF, N. A. G.; VERGINELLI, G.; JATENE, A. D. - Plástica da valva mitral em pacientes consecutivos. Como é a evolução tardia?: avaliação clínica e ecocardiográfica. Rev. Bras. Cir. Cardiovasc., 6(2): 63-79, 1992. of bovine pericardium used in cardiac valve bioprotheses: implantations for the mechanisms of bioprosthetic tissue mineralization. Am. J. Pathol., 123: 134-145.

SHERRID, M. V.; CLARK, R. D.; COHN, K. - Echocardiographic analysus of left atrial size before and after operation in mitral valve disease. Am. J. Cardiol., 43: $171-178,1979$

94 SHORE, D. F.; GABBAY, S.; YELLIN, E. L.; FRATER, R. W. M. - Degenerative changes in glutaraledehyde preserved pericardium used for the experimental replacement of anterior chordal of mitral valve. J. Cardiovasc. Surg., 24: 132-137, 1983.

SHORE, D. F.; WONG, P.; PANETH, M. - Valve repair versus replacement in the surgical management of ruptured chordial. A post-operative echocardiographic assessment of mitral valve function. J. Cardiovasc. Surg., 23: 378-382, 1982.

SHORE, D. F.; WONG, P.; PENETH, M.; BUCKLEI, M. J. - Results of mitral valvuloplasty with a suture pication technique. J. Thorac. Cardiovasc. Surg., 79: 349-357, 1980.

SIGGAARD-ANDERSEN, O. - Blood acid-base alignment nomogram-scales for $\mathrm{pH}, \mathrm{pC002}$, base excess of whole blood of different hemoglobin cncentrations plasma bicarbonate and plasma total- $\mathrm{CO2}$. Scand. J. Clin. Lab. Invest., 15: 211, 1963.

SPENCER, F. C.; COLVIN, S. B.; CULLIFORD, A. T.; ISON, O. W. - Experience with the Carpentier technique of mitral valve reconstruction in 103 patients (1980-1985). J. Thorac. Cardiovasc. Surg., 90: $341-350,1985$.

SYRACUSE, D. C.; BOWMAN Jr., Fo.; MALM. J. R. Prosthetic valve reoperations: factors influencing early and late survival. J. Thorac. Cardiovasc. Surg., 77: 346-353, 1979.

WEINSTEIN. I. R.; MARBARGER, J. P.; PÉREZ, J. E. - Ultrasonic assessment of the St. Jude prosthetic valve: $\mathrm{M}$ mode, two-dimensional, and Doppler echocardiography. Circulation, 68: 897-905, 1983.
104 WILLIAMS, G. A. \& LABOVITZ, A. J. - Doppler hemodynamic evaluation of prosthetic (Starr-Edwards and Bjürk-Shiley) and bioprosthetic (Hancok and Carpentier-Edwards) cardiac valves. $A m$. J. Cardiol., f56: 325-332, 1985.

105 WONG, C. Y. H. \& SPIOTNITZ, H. M. - Systolic and diastolic properties of the human left ventricle during valve replacement for chronic mitral regurgitation. Am. J. Cardiol., 47: 40-50, 1981

106 YACOUB, M.; HALIM, M.; RADLEY-SMITH, R.; MCKAY, R.; NIJVELD, A.; TOWERS, M. - Surgical treatment of mitral regurgiration caused by floppy valves: repair vesus replacement. Circulation, 64 (Supl. 2): 210-216, 1981.

107 YANG, S. S.; BENTIVOGLIO, L. G.; MARANHÃO, V.; GOLDBERG, H. - Assessment of ventricular funcition. In: From cardiac catheterization data to hemodynamic parameters. 3. ed. Philadelphia, F. A. Daris Company, 1988. Cap. 10, p. 189-255.

108 ZWENG, T. N.; BLUETT, M. K.; MOSCA, R.; CALLOW, L. B.; BOVE, E. L. - Mitral valve replacement in the first 5 years of life. Ann. Thorac. Surg., 47: 720-724, 1989.

\section{Discussão}

\section{DR. FRANCISCO GREGORI Jr.} Londrina, $P R$

Quero, inicialmente, parabenizar os autores, pelo excelente estudo, o que justifica a brilhante defesa de tese realizada pelo Dr. Pablo. Assim como o Dr. Pablo temo-nos dedicado ao tratamento reparador da valva mitral insuficiente. Entre junho e 1979 e setembro de 1987, realizamos 108 plásticas da valva mitral empregando anel de Carpentier. Setenta e oito por cento dos pacientes eram reumáticos, com idade média de 37 anos. A mortalidade imediata foi de 1,9\% (2 casos) e tardia $1,9 \%$ (2) com $16,7 \%$ (18) de reoperação. Apenas um paciente apresentou acidente tromboembólico. A partir de 1987, passamos a empregar anéis abertos Gregori-IMC, tendo sido realizadas, até os dias atuais, 60 anuloplastias. Noventa por cento dos pacientes eram reumáticos, com idade média de 25 anos, sendo 24 $(40,7 \%)$ com idade inferior a 16 anos, com tempo médio de seguimento de 18 meses. Não tivemos óbitos hospitalares ou tardios neste grupo. Seis $(10 \%)$ pacientes foram reoperados, tendo sido realizada nova plástica em dois e troca valvar em quatro. As causas da insuficiência mitral pós-operatória foram a reativaçāo reumática (3), a endocardite infecciosa (2) e a calcificaçăo (1). Não aconteceram fenômenos tromboembólicos. Nos dois grupos, procedimentos associados sobre as cordas tendíneas, especialmente o encurtamento de cordas alongadas, foram realizados em mais de $60 \%$ dos pacientes. Achamos baixo o índice de atuaçăo sobre as cordas 
POMERANTZEFF, P. M. A.; AZEVEDO, J. G.; RATTI, M.; MORAES, A. V.; AMATO, M.; GRINBERG, M.; MONTEIRO, A. C.; PUIG, L. B.; STOLF, N. A. G.; VERGINELLI, G.; JATENE, A. D. - Plástica da valva mitral em pacientes consecutivos. Como é a evolução tardia?: avaliação clínica e ecocardiográfica. Rev. Bras. Cir. Cardiovasc., 6(2): 63-79, 1992.

tendíneas no seu grupo (13\%). Qual é a explicação? Também achamos baixo o índice de pacientes com ausência de sopros mitrais $(28 \%)$ para um tempo médio de pós-operatório de 39 meses. Na nossa casuística, mais de $60 \%$ dos casos estāo sem sopros e $30 \%$ com sopros sistólicos discretos na análise global. Os índices favorecem o grupo com anel aberto, porém o tempo de segmento é muito maior no grupo com anéis de Carpentier. Mesmo assim, aproximadamente, $50 \%$ dos pacientes deste grupo estão sem sopros com tempo médio de seguimento de 92 meses. Poderia haver alguma relação entre o baixo índice de atuação sobre as cordas tendineas nos seus casos com o alto índice de pacientes com sopro sistólico mitral, mesmo que discreto? No estudo ecodoplercardiográfico, o Dr. Pablo encontrou gradiente mitral pós-operatório de $5,1 \mathrm{mmHg}$ em repouso e $9 \mathrm{mmHg}$ ao exercício. Pergunto se houve diferença entre o grupo com anel de Carpentier e com fita posterior, uma vez que, em nosso material, encontramos 6,3 $\mathrm{mmHg}$ no grupo com anel de Carpentier e $2,9 \mathrm{mmHg}$ com anel aberto e atribuímos este fato ao caráter não constritivo dos anéis abertos. Finalmente, pediria que o Dr. Pablo fizesse uma análise crítica comparativa dos anéis de Carpentier e da fita posterior e nos dissesse quando emprega uma ou outra técnica. Aproveito a oportunidade para perguntar se tem utilizado anéis de terceira geração como chamou com muito entusiasmo os de Puig Massana e Abdulmassih, no último Congresso realizado em São Paulo. Se não, por que?

\section{DR. POMERANTZEFF \\ (Encerrando)}

Agradeço os comentários do Dr. Gregori Jr., cirurgião que, como todos nós sabemos, se dedica, há muito tempo, às plastias valvares. No material que o Dr. Gregori apresentou, não houve mortalidade hospitalar, o que coincide com a nossa experiência, neste estudo e conforme a literatura internacional, que também demonstra menor risco para os pacientes, em relação à troca valvar mitral. Com relação ao baixo índice de encurtamento de cordas em nosso material, gostaríamos de salientar que estes foram os nossos primeiros casos, e que, atualmente, nos pacientes reumáticos e isquêmicos, temos realizado este procedimento para proporcionar melhor cooptação das cúspides, pretendendo, desta forma, meIhor evolução a longo prazo. A nossa incidência de sopros é comparável com vários relatos da literatura, como, por exemplo, Yacoub, porém acredito que o tipo de valva que estamos consevando, isto é, valvas mais degeneradas, tem relação com a porcentagem de sopro residual. O esforço para preservar valvas ruins em pacientes jovens acarreta piores resultados. Além disso, a curva de aprendizado em todos os grupos que fazem plástica é evidente. Em nosso material, não encontramos diferença significante de gradiente ao exercício, nos pacientes operados com a técnica de fita posterior e anel de Carpentier; porém acredito ser este um ponto muito importante nas plásticas mitrais, que é a preocupação com a área valvar, no final da plastia. Tenho utilizado o anel de Carpentier quando o diâmetro vertical da mitral, isto é, o diâmetro entre o fluxo médio da cúspide anterior e o ponto médio da cúspide posterior está muito aumentado, o que significa uma grande deformidade do anel mitral. Não tenho utilizado anéis ditos de terceira geração, pois não concordo com a filosofia de anéis que não moldam o anel mitral. Antes de terminar gostaria de agradecer, mais uma vez, o critérioso comentário do nosso amigo e excelente cirurgiāo, Francisco Gregori, e salienter a importância que ocupam atualmente, as plásticas de valva mitral, e chamamos a atenção, para os resultados das plásticas em pacientes com degeneração mixomatosa. Muito obrigado. 